NONSTANDARD HULLS OF ORDERED VECTOR SPACES

\author{
A THESIS SUBMITTED TO \\ THE GRADUATE SCHOOL OF NATURAL AND APPLIED SCIENCES \\ $\mathrm{OF}$ \\ MIDDLE EAST TECHNICAL UNIVERSITY
}

BY

HASAN GÜL

IN PARTIAL FULFILLMENT OF THE REQUIREMENTS

FOR

THE DEGREE OF DOCTOR OF PHILOSOPHY

IN

MATHEMATICS

DECEMBER 2015 

Approval of the thesis:

\section{NONSTANDARD HULLS OF ORDERED VECTOR SPACES}

submitted by HASAN GÜL in partial fulfillment of the requirements for the degree of Doctor of Philosophy in Mathematics Department, Middle East Technical University by,

Prof. Dr. Gülbin Dural Ünver

Dean, Graduate School of Natural and Applied Sciences

Prof. Dr. Mustafa Korkmaz

Head of Department, Mathematics

Prof. Dr. Eduard Emelyanov

Supervisor, Mathematics Dept., METU

\section{Examining Committee Members :}

Prof. Dr. Zafer Nurlu

Mathematics Dept., METU

Prof. Dr. Eduard Emelyanov

Mathematics Dept., METU

Prof. Dr. Bahri Turan

Mathematics Dept., Gazi University

Prof. Dr. Birol Altın

Mathematics Dept., Gazi University

Assist. Prof. Dr. Konstyantyn Zheltukhin

Mathematics Dept., METU 
I hereby declare that all information in this document has been obtained and presented in accordance with academic rules and ethical conduct. I also declare that, as required by these rules and conduct, I have fully cited and referenced all material and results that are not original to this work.

Name, Last name: Hasan Gül

Signature: 


\title{
ABSTRACT \\ NONSTANDARD HULLS OF ORDERED VECTOR SPACES
}

\author{
Gül, Hasan
}

Ph. D., Department of Mathematics

Supervisor : Prof. Dr. Eduard Emelyanov

\begin{abstract}
December 2015, 57 pages
This thesis undertakes the investigation of ordered vector spaces by applying nonstandard analysis. We introduce and study two types of nonstandard hulls of ordered vector spaces. Norm-nonstandard hulls of ordered Banach spaces are also investigated
\end{abstract}

MSC: 46A40, 46B40

Key words: ordered vector space, Krein space, nonstandard extension, nonstandard hull 
$\ddot{O Z Z}$

\title{
NONSTANDARD HULLS OF ORDERED VECTOR SPACES
}

\author{
Gül, Hasan \\ Doktora, Matematik Bölümü \\ Tez Yöneticisi : Prof. Dr. Eduard Emelyanov
}

Aralık 2015, 57 sayfa

$\mathrm{Bu}$ tezde standart olmayan analiz yöntemleri ile sıralamalı vektör uzayların incelenmesine başlanacaktır. Sıralamalı vektör uzaylarına ait iki tür standart olmayan zarflar tanımlanıp araştırılacaktır. Sıralamalı Banach uzaylarının norm standart olmayan zarfları da ayrıca incelenecektir.

MSC : 46A40, 46B40

Anahtar Kelimeler : siralamalı vector uzay1, Krein uzayı, standart olmayan genişleme, standart olmayan zarf 


\section{ACKNOWLEDGEMENTS}

I would like to express my gratitude to my former advisors, Prof. Dr. Süleyman Önal and Prof. Dr. Şafak Alpay for extending me positive stimuli and my current advisor Prof. Dr. Eduard Emelyanov for introducing me to the joys of nonstandard analysis.

I am also obliged to my thesis supervisory committee members, Prof. Dr. Bahri Turan, Prof. Dr. Ayşe Uyar and Prof. Dr. Birol Altın for their valuable comments.

I acknowledge the faculty members and staff of the Department of Mathematics in my studies for their contributions in my studies.

Lastly, but not in the least, I am indebted to my family for their continuing support. 


\section{TABLE OF CONTENTS}

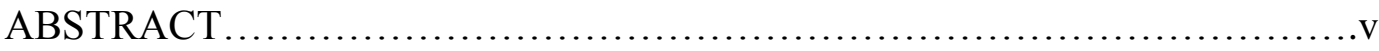

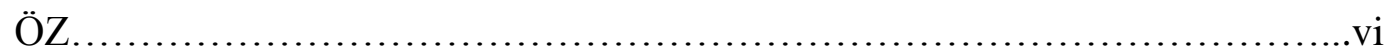

ACKNOWLEDGEMENTS..............................................vii

TABLE OF CONTENTS ........................................,,,,,.,viii

CHAPTERS

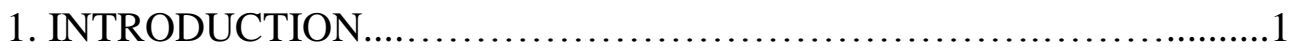

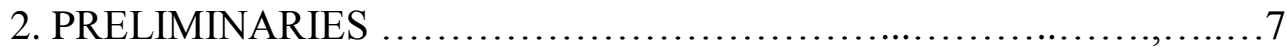

2.1 Nonstandard Extensions $\ldots \ldots \ldots \ldots, \ldots \ldots \ldots \ldots \ldots \ldots \ldots \ldots \ldots \ldots \ldots, . .7$

2.2 Ordered Vector Spaces $\ldots \ldots \ldots \ldots \ldots \ldots \ldots \ldots \ldots \ldots \ldots \ldots \ldots \ldots, \ldots, \ldots$

2.3 Nonstandard Hulls .............................................13

3. SOME EXTERNAL VECTOR SPACES ASSOCIATED WITH

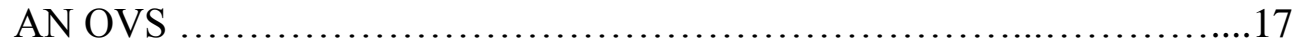

4. THE ORDER AND THE REGULAR NONSTANDARD HULLS

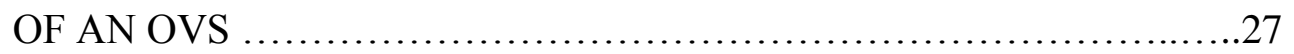

5. NORMED OVS ............................................. 31

6. LINEAR OPERATORS BETWEEN OVS .........................43

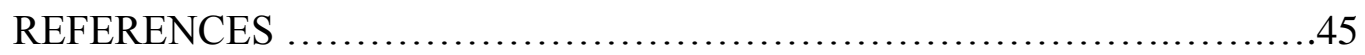

APPENDICES

A. THE ULTRAPOWER CONSTRUCTION OF NONSTANDARD

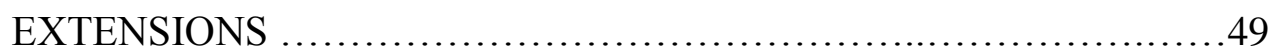



viii 


\section{CHAPTER 1}

\section{INTRODUCTION}

Nonstandard Analysis may seem, at first glance, a rigorous revitalization of naive utilization of infinitesimal and infinite elements by mathematicians till Cauchy and Weierstrauss, that is, $\varepsilon, \delta$ formalism, after the works of Skolem (1934) and Robinson (1966). But, one may claim that "... one should be careful in claiming that novel developments prove the correctness of older ideas." [1]

The basic idea is a construction of a proper extension of $\mathbb{R}$, denoted by ${ }^{*} \mathbb{R}$, in which all of the properties of the ordered field $\mathbb{R}$ is conserved in a sense, which will be made explicit later.

Of course, the construction of nonstandard extension ${ }^{*} \mathbb{R}$ of $\mathbb{R}$ may be generalized to construct a nonstandard extension ${ }^{*} X$ of any algeabraic structure $X$.

The list of applications of nonstandard analysis, which proved to give us extremely powerful mathematical tools, such as the hyperfinite approximation, Loeb Measure ( which is employed in applications of nonstandard analysis to probability theory, stochastic analysis and mathematical finance) is significantly large in the functional analysis (see, for example, $[1,17,18,20])$.

One of the most important constructions while studying local properties of locally convex vector spaces is of the nonstandard hull of a normed space by Luxemburg [21].

But, first, consider the following sets.

(1) The set of finite hyperreals is

$$
\operatorname{Fin}\left({ }^{*} \mathbb{R}\right):=\left\{x \in{ }^{*} \mathbb{R}:|x| \leqslant n \text { for some } \mathrm{n} \in \mathbb{N}\right\}
$$


(2) The set of infinite hyperreals is ${ }^{*} \mathbb{R}_{\text {inf }}:={ }^{*} \mathbb{R} \backslash \operatorname{Fin}\left({ }^{*} \mathbb{R}\right)$

(3) The set of infinitesimal hyperreals is

$$
\mu:=\left\{x \in{ }^{*} \mathbb{R}:|x| \leqslant \frac{1}{n} \text { for all } \mathrm{n} \in \mathbb{N}_{+}\right\}
$$

Note that $\mu \cap \mathbb{R}=\emptyset, \operatorname{Fin}\left({ }^{*} \mathbb{R}\right)$ is a subring of ${ }^{*} \mathbb{R}$, and $\mu$ is an ideal of $\operatorname{Fin}\left({ }^{*} \mathbb{R}\right)$. For $x, y \in{ }^{*} \mathbb{R}$, we say $x$ and $y$ are infinitely close, denote by $x \approx y$, if $x-y \in \mu$. If $r \in \operatorname{Fin}\left({ }^{*} \mathbb{R}\right)$ then there is a unique $s \in \mathbb{R}$ such that $r \approx s$. We call s standard part of $r$ and write $s t(r)=s$. Clearly, st $:$ Fin $\left({ }^{*} \mathbb{R}\right) \rightarrow \mathbb{R}$ is a surjective ring homomorphism. Thus, $\operatorname{Fin}\left({ }^{*} \mathbb{R}\right) / \mu \cong \mathbb{R}$ is our, somewhat trivial, first nonstandard hull construction. Note that if we start with $\mathbb{Q}$, we will end up with $\operatorname{Fin}\left({ }^{*} \mathbb{Q}\right) / \mu(* \mathbb{Q}) \cong \mathbb{R}$.

Now let us return to Luxemburg's elegant construction. Let $X=(X,\|\cdot\|)$ be a normed space. It can be shown that ${ }^{*} X$ is a vector space over $\mathbb{R}$. Consider also the following vector spaces over $\mathbb{R}$. The space

$$
\operatorname{Fin}\left({ }^{*} X\right):=\left\{\kappa \in{ }^{*} X:(\exists M \in \mathbb{R})\|\kappa\| \leq M\right\}
$$

of norm-finite elements of ${ }^{*} X$ and the space

$$
\mu\left({ }^{*} X\right):=\left\{\kappa \in{ }^{*} X:(\exists M \in \mathbb{R})(\forall n \in \mathbb{N})\|n \kappa\| \leq M\right\}
$$

of norm-infinitesimal elements $(\|\cdot\|$-infinitesimals $)$ of ${ }^{*} X$.

Note that $\mu\left({ }^{*} X\right)$ is a subspace of semi-normed space $\left(\operatorname{Fin}\left({ }^{*} X\right), \operatorname{st}(\|\cdot\|)\right)$ and $\mu\left({ }^{*} X\right)=\operatorname{ker}(\operatorname{st}(\|\cdot\|))$. Then the quotient $\tilde{X}:=\operatorname{Fin}\left({ }^{*} X\right) / \mu\left({ }^{*} X\right)$ is a Banach space, under the norm $\|[\kappa]\|=\operatorname{st}(\|\kappa\|)$. We call the (external) normed space $\tilde{X}:=(\tilde{X},\|\cdot\|)$ together with the natural embedding

$$
X \stackrel{*}{\rightarrow} \operatorname{Fin}\left({ }^{*} X\right) \stackrel{i}{\rightarrow} \tilde{X}
$$


the norm-nonstandard hull of $X$. In this thesis, we use slightly different name for $\tilde{X}$, which is commonly known as nonstandard hull of $X[1,17,7]$. The reason lies in the fact that the same vector space $X$ may simultaneously carry several different structures together with the norm structure and, hence, $X$ may possess several different types of nonstandard hulls together with the norm-nonstandard hull. There is a well developed theory of norm-nonstandard hulls of normed spaces and operators between them (see, e.g., [17]).

In the case when $X$ is a vector lattice, no norm is available in general. This reason has motivated the study of different kinds of nonstandard hulls of $X$ (see, e.g., $[9,7]$ ).

Emel'yanov investigated infinitesimal approach to vector lattices in the early 1990's $[8,10,11,12,13,16,14]$ (for survey of these results see $[8,7]$ ). For instance, in papers $[12,13,14]$ two different types of nonstandard hulls of vector lattices were introduced and studied. Then, in [11] a modified method of construction of nonstandard hulls has been applied to lattice normed vector spaces and dominated operators between them.

Now that nonstandard hulls of vector lattices are sufficiently well explored, it is natural to employ methods of nonstandard analysis to investigate a class of vector spaces more general than vector lattices, namely the vector spaces with positive cones, which are known as ordered vector spaces (OVS, in short). Recently, the investigation of OVS $[5,6]$ has been carried out and we initiated the study of nonstandard hulls in this framework.

In this thesis, we introduce and study nonstandard hulls of OVS and of operators between them. More specifically, after the preliminaries , we study some several external vector spaces associated with OVS in Chapter 3. This chapter can be considered as a continuation of development of nonstandard tools for OVS, started in $[5,6]$. 
Consider the following external sets: the set

$$
\operatorname{fin}\left({ }^{*} X\right):=\left\{\kappa \in{ }^{*} X:(\exists x, y \in X) \kappa \in[x, y]\right\}
$$

of order-finite elements of ${ }^{*} X$, the set

$$
\lambda\left({ }^{*} X\right):=\left\{\kappa \in{ }^{*} X:(\exists y \in X)(\forall n \in \mathbb{N}) n \kappa \in[-y, y]\right\}
$$

of regular-infinitesimal elements ( $r$-infinitesimals) of ${ }^{*} X$, the set

$$
\eta\left({ }^{*} X\right):=\left\{\kappa \in{ }^{*} X: \inf _{X} U(\kappa)=\sup _{X} L(\kappa)=0\right\}
$$

of order-infinitesimal elements (o-infinitesimals) of ${ }^{*} X$.

The sets fin $\left({ }^{*} X\right), \lambda\left({ }^{*} X\right), \eta\left({ }^{*} X\right)$ are ordered vector spaces over $\mathbb{R}$ with respect to the ordering inherited from ${ }^{*} X$. Moreover, $\lambda\left({ }^{*} X\right)$ and $\eta\left({ }^{*} X\right)$ are order ideals in fin $\left({ }^{*} X\right)$.

In Chapter 4, we introduce order and regular nonstandard hulls of OVS and study their elementary properties. Define

$$
{ }_{(o)} X:=\operatorname{fin}\left({ }^{*} X\right) / \eta\left({ }^{*} X\right) .
$$

The mapping $X \stackrel{\hat{\eta}^{\rightarrow}}{\rightarrow}(o)$ defined by $\hat{\eta}(x)=\left[{ }^{*} x\right]$ is an order embedding since $\eta\left({ }^{*} X\right) \cap X=$ $\{0\}$. OVS ${ }_{(o)} X$ is called the order (nonstandard) hull of $X$. Consider the following quotient space with respect to the canonical partial ordering:

$$
(r) X:=\operatorname{fin}\left({ }^{*} X\right) / \lambda\left({ }^{*} X\right)
$$

The corresponding order homomorphism $X \stackrel{\hat{\lambda}}{\rightarrow}{ }_{(r)} X, \lambda(x)=\left[{ }^{*} x\right]$, is an order embedding iff $\lambda\left({ }^{*} X\right) \cap X=\{0\}$, and, by Theorem 4, iff $X$ is almost Archimedean. For an almost Archimedean OVS $X$, the OVS ${ }_{(r)} X$ is called the regular (nonstandard) hull of $X$. 
In Chapter 5, we turn our attention to Luxemburg's nonstandard hull of a normed OVS and discuss some results in connection with the the normality of the positive cone.

In Chapter 6, we start to investigate linear operators from a nonstandard point of view.

We do hope that the techniques that are developed here, may serve in the theory of OVS by providing new outlook at some of the old problems in there. Also, conversely, the theory of nonstandard hulls of OVS possesses many interesting, difficult, and still unsolved problems. 


\section{CHAPTER 2}

\section{PRELIMINARIES}

\subsection{Nonstandard extensions}

There are two approaches to construct a nonstandard extension which satisfy our requirements, one that utilizes the compactness theorem and the other, the ultrapower construction which is outlined in the Appendix. The approach to the nonstandard analysis that we use follows that of Stroyan and Luxemburg [21] .

Let $\mathrm{S}$ be a set . A superstructure over $\mathrm{S}$ is the set $V(S):=\bigcup_{n} V_{n}(S)$, with $V_{n}(S)$ defined by recursion:

$$
\begin{gathered}
V_{1}(S):=S, \\
V_{n+1}(S):=\mathscr{P}\left(V_{n}(S)\right), \\
V(S):=\bigcup_{n} V_{n}(S),
\end{gathered}
$$

where $\mathscr{P}(X)$ denotes the power set of $\mathrm{X}$ and $\mathrm{S}$ is a set often not specified explicitly but chosen large enough to contain all objects under consideration, real numbers, vector spaces, sets of functions, etc. 
We may suppose that for the extension ${ }^{*} S$ of the set $S$, the natural embedding

$$
*: V(S) \hookrightarrow V\left({ }^{*} S\right)
$$

satisfies the following principles :

Extension Principle. The set $S$ is a proper subset of ${ }^{*} S$ (assuming ${ }^{*} x=x$ for every $x \in S)$. Moreover, ${ }^{*} S$ is equipped with the same operations and relations as $S$ is.

Transfer Principle. Let $\psi\left(x_{1}, x_{2}, \ldots, x_{n}\right)$ be a bounded formula of the superstructure $V(S)$ (i.e., a formula of the restricted language $L(V(S))[1, \mathrm{p} .18]$ ), and let $A_{1}, A_{2}, \ldots, A_{n}$ be elements of the superstructure $V(S)$.

Then the assertion $\psi\left(A_{1}, A_{2}, \ldots, A_{n}\right)$ about elements of $V(S)$ holds true iff the assertion $\psi\left({ }^{*} A_{1},{ }^{*} A_{2}, \ldots,{ }^{*} A_{n}\right)$ about elements of ${ }^{*}(V(S))$ does.

Let $*(V(S))$ be a nonstandard enlargement of a superstructure $V(S)$. An element $x \in{ }^{*}(V(S))$ is called: standard if $x={ }^{*} X$ for some $X \in V(S)$; internal if $x \in{ }^{*} X$ for some $X \in V(S)$; external if $x$ is not internal. Note that every standard element is internal, and every element of an internal set is internal .

Internal Definition Principle. Given a formula $\psi\left(x, x_{1}, \ldots, x_{n}\right)$ of $L(V(S))$ and internal sets $A, A_{1}, \ldots, A_{n}$. Then the set $\left\{x \in A: \psi\left(x, A_{1}, \ldots, A_{n}\right)\right\}$ is internal.

General Saturation Principle. If a family $\left\{A_{\gamma}\right\}_{\gamma \in \Gamma}$ of internal sets possesses the finite intersection property and $\operatorname{card}(\Gamma)<\operatorname{card}(V(S))$, then $\bigcap_{\gamma \in \Gamma} A_{\gamma} \neq \emptyset$.

Lemma 1. For every directed set $(\Theta, \prec) \in V(S)$, there is an infinitely remote element $a \in{ }^{a} \Theta:=\left\{\xi \in{ }^{*} \Theta:(\forall \tau \in \Theta) \tau \prec \xi\right\}$. 
One of equivalent forms of Lemma 1 is the following: in a polysaturated enlargement of $V(S)$, any set $A$ with $\operatorname{card}(A)<\operatorname{card}(V(S))$ is a subset of a hyperfinite set $B$ (meaning that there is an internal bijection between $B$ and some $v \in{ }^{*} \mathbb{N}$ ).

\subsection{Ordered vector spaces}

All vector spaces under consideration in this thesis assumed to be over the real field $\mathbb{R}$. A subset $K$ of a vector space $X$ is called a cone if it satisfies

$$
K \cap(-K)=\{0\}, K+K \subseteq K \text { and } r K \subseteq K
$$

for all $r \geq 0$. A cone $K$ is said to be generating if $K-K=X$. Given a cone $X_{+}$in $X$, we say that $\left(X, X_{+}\right)$is an ordered vector space. The partial ordering $\leq$on $X$ is defined by

$$
x \leq y \text { if } y-x \in X_{+} .
$$

The OVS $\left(X, X_{+}\right)$is also denoted by $(X, \leq)$ or simply by $X$ if the partial ordering is well understood.

For every $x, y \in X$, the (possibly empty if $x \not \leq y$ ) set

$$
[x, y]=\{z: x \leq z \leq y\}
$$

is called an order interval. A vector subspace $I$ of $X$ is called an order ideal if for every $x, y \in I$ we have that $[x, y] \subset I$. It is well known that if $Y \subseteq X$ is an order ideal, then the quotient space $X / Y$ is an OVS with respect to the following ordering:

$$
[0] \leq[f] \text { if } \exists q \in Y \text { with } 0 \leq f+q
$$

An OVS $X$ is said to be Archimedean, if

$$
\left[(\forall n \geq 1) n y \leq x \in X_{+}\right] \Rightarrow[y \leq 0]
$$


Note that an $x \in X_{+}$in the definition above can be replaced by an arbitrary $x \in X$.

Also, $\mathrm{X}$ is Archimedean iff $\inf _{n \geq 1} \frac{1}{n} x=0$ for all $x \in X_{+}$.

One may say that an Archimedean space does not contain any infinitely small or large comparable elements.

An OVS $X$ is called almost Archimedean if

$$
\left[(\forall n \in \mathbb{Z}) n y \leq x \in X_{+}\right] \Rightarrow[y=0]
$$

for every $x \in X_{+}$. Clearly, $X$ is almost Archimedean iff

$$
\bigcap_{n \geq 1}\left[-\frac{1}{n} x, \frac{1}{n} x\right]=\{0\} \quad \forall x \in X_{+} .
$$

Archimedean ordered vector spaces are are almost Archimedean but the converse does not hold.

Example 1. Let $\mathrm{X}$ be the ovs with generating cone

$$
\begin{gathered}
X_{+}=\left\{(x, y) \in \mathbb{R}^{2} \mid x>0 \text { and } y \geq 0 \text { and or } x=y=0\right\} \\
n(0,-1) \leq(1,1)
\end{gathered}
$$

holds for all $n \in \mathbb{N}$, but $(0,-1) \nless(0,0)$. Thus, $\left(X, X_{+}\right)$is not Archimedean. However, $\left(X, X_{+}\right)$is almost Archimedean for it is a subcone of an almost Archimedean cone given by $\left\{(x, y) \in \mathbb{R}^{2} \mid x \geq 0\right.$ and $\left.y \geq 0\right\}$.

Example 2.The Euclidean plane with lexicographic ordering $\left(\mathbb{R}^{2}, \leq_{\text {lex }}\right)$ is not almost Archimedean.

$X_{+}=\left\{(x, y) \in \mathbb{R}^{2} \mid x>0\right.$ or $x=0$ and $\left.y \geq 0\right\}$ is the generating cone. $n(0,1) \leq(1,0)$ holds for all $n \in \mathbb{Z}$, but $(0,1) \neq(0,0)$. 
An OVS $X$ is said to be order complete if every nonempty bounded above subset of $X$ has a supremum (cf. [26, p.209]). $X$ is called Dedekind complete if every increasing bounded above net in $X$ has a supremum [2]. It is easy to see that any order complete OVS is Dedekind complete, but, in general, not vice versa.

Let $\left\{x_{\xi}\right\}$ be a net in $X$. Then $\left\{x_{\xi}\right\}$ is said to be order convergent to an $x \in X$ (in symbols $x_{\xi} \stackrel{o}{\rightarrow} x$ ), if there exists a net $\left\{p_{\xi}\right\} \downarrow 0$ in $X_{+}$such that

$$
x_{\xi}-x \in\left[-p_{\xi}, p_{\xi}\right]
$$

Let $\left(x_{n}\right)$ be a sequence in $X$ and $u \in X_{+}$, then $\left(x_{n}\right)$ is said to be $u$-uniformly convergent to an $x \in X$ (in symbols $x_{n} \stackrel{u}{\rightarrow} x$ ), if

$$
x_{n}-x \in\left[-\varepsilon_{n} u, \varepsilon_{n} u\right]
$$

for some sequence $\varepsilon_{n}$ of reals such that $\varepsilon_{n} \downarrow 0$, or, equivalently, for any given $\varepsilon>0$, there exists $n(\varepsilon) \in \mathbb{N}$ such that

$$
x_{n}-x \in[-\varepsilon u, \varepsilon u]
$$

for all $n \geqslant n(\varepsilon)$. In general, these u-uniform limits may not be unique.

Example 3. Consider the sequence of the lexicographic plane ( a non-Archimedean space) defined by $x_{n}=\left(\frac{1}{n}, 0\right)$ and $u=(2,2)$. Then for every vector of the form $x=(0, r)$, where $r \in \mathbb{R}$, we have

$$
x_{n}-x=\left(\frac{1}{n}, 0\right)-(0, r)=\left(\frac{1}{n},-r\right) .
$$

Since $(0,0) \leq\left(\frac{1}{n},-r\right) \leq\left(\frac{2}{n}, \frac{2}{n}\right)$, we have $0 \leq x_{n}-x \leq \frac{1}{n} u$, that is, each vector of the form $(0, r)$ is a limit for each $r \in \mathbb{R}$. 
- The uniform limits are unique in an Archimedean OVS.

Proof: Suppose a sequence $\left(x_{n}\right)$ in an Archimedean OVS X satisfies $x_{n} \stackrel{u}{\rightarrow} x$ and $x_{n} \stackrel{v}{\rightarrow} y$. for some $u, v \in X_{+}$. Fix $k$ and choose $n$ such that $-\frac{1}{k} u \leq x_{n}-x \leq \frac{1}{k} u$ and $-\frac{1}{k} v \leq y-x_{n} \leq \frac{1}{k} v$. Adding the inequalities yields

$$
-\frac{1}{k}(u+v) \leq y-x \leq \frac{1}{k}(u+v)
$$

for each $k$. Since $X$ is Archimedean, $y-x=0$ or $y=x$.

- Let $\left(x_{n}\right)$ be a sequence in an Archimedean OVS $X$. If $\left(x_{n}\right)$ converges uniformly, then it is also convergent in order. But, in general, order convergence of $\left(x_{n}\right)$ does not imply relative uniform convergence.

Proof: Suppose $x_{n} \stackrel{u}{\rightarrow} x$, that is, there exists $u \in X_{+}$and a sequence of reals $\varepsilon_{n} \downarrow 0$ such that $-\varepsilon_{n} u \leq x_{n}-x \leq \varepsilon_{n} u$ for all $n \in \mathbb{N}$. Since $X$ is Archimedean, $\varepsilon_{n} u \downarrow 0$. Then, let $y_{n}=\varepsilon_{n} u$ for each $n \in \mathbb{N}$ to claim that $\left(x_{n}\right)$ converges in order to $x$.

Example 4. Let $X$ be the Archimedean space $1_{\infty}$ of all bounded real sequences with coordinatewise ordering and $x_{n}$ be the element with first $\mathrm{n}$ coordinates equal to 0 , and the rest coordinates equal to 1 . Then, $x_{n} \downarrow 0$ in order, but there does not exist any $u \in\left(1_{\infty}\right)_{+}$to be a regulator for the relative uniform convergence of $\left(x_{n}\right)$.

The positive cone $X_{+}$in a normed $\mathrm{OVS} X$ is said to be normal if there exists $\varepsilon>0$ such that for every $x, y \in X_{+}$with $\|x\|=\|y\|=1$ we have $\|x+y\| \geq \varepsilon$. It can be shown that $X_{+}$is normal iff $X$ admits an equivalent monotone norm $\|\cdot\|_{m}$ (that is: $0 \leq x \leq y$ implies $\|x\|_{m} \leq\|y\|_{m}$ ) and iff every order bounded set is bounded in norm (that is, normality is a condition of compatibility of the order and the topology).

Example 5. $C^{1}[0,1]$ does not have a normal cone under the norm $\|f\|=\|f\|_{\infty}+\|$ $f^{\prime} \|_{\infty}$ 
The norm $\|\cdot\|$ in $X$ is said to be order continuous if for every net $x_{\xi}$ in $X, x_{\xi} \downarrow 0$ implies $\left\|x_{\xi}\right\| \downarrow 0$.

An element $e$ of an OVS $X$ is called an order unit if for every $x \in X$ there exists $n \in \mathbb{N}$ such that $-n e \leqslant x \leqslant n e$.

A Banach OVS $\left(X, X_{+},\|\cdot\|\right)$ with the closed positive cone $X_{+}$and an order unit is called a Krein space. The positive cone of a Krein space need not be normal (see Example 5).

The positive cone $X_{+}$in a Banach OVS $X$ satisfies the Levi property (or an ordered Banach space $\left(X, X_{+},\|\cdot\|\right)$ has the Levi property) if it follows from $0 \leq x_{n} \uparrow \leq x$ that the sequence $x_{n}$ is norm convergent in $\mathrm{X}$.

An OVS $X$ is said to be vector lattice if every nonempty finite subset of $X$ has a supremum. It is well known that any almost Archimedean vector lattice is Archimedean and any Dedekind complete vector lattice is order complete. For further information on ordered spaces we refer to $[2,22,20,26,28]$.

\subsection{Nonstandard hulls}

Let $X=\left(X,\|\cdot\|\right.$ be a normed space. Clearly, ${ }^{*} X$ is a vector space over $\mathbb{R}$. Consider also the following vector spaces over $\mathbb{R}$. The space

$$
\operatorname{Fin}\left({ }^{*} X\right):=\left\{\kappa \in{ }^{*} X:(\exists M \in \mathbb{R})\|\kappa\| \leq M\right\}
$$

of norm-finite elements of ${ }^{*} X$ and the space

$$
\mu\left({ }^{*} X\right):=\left\{\kappa \in{ }^{*} X:(\exists M \in \mathbb{R})(\forall n \in \mathbb{N})\|n \kappa\| \leq M\right\}
$$


of norm-infinitesimal elements $(\|\cdot\|$-infinitesimals $)$ of ${ }^{*} X$. The following elegant construction was invented by Luxemburg [21].

Note that $\mu\left({ }^{*} X\right)$ is a subspace of semi-normed space $\left(\operatorname{Fin}\left({ }^{*} X\right), \operatorname{st}(\|\cdot\|)\right)$ and $\mu\left({ }^{*} X\right)=\operatorname{ker}(\operatorname{st}(\|\cdot\|))$. Then the quotient $\tilde{X}:=\operatorname{Fin}\left({ }^{*} X\right) / \mu\left({ }^{*} X\right)$ is a Banach space, under the norm $\|[\kappa]\|=\operatorname{st}(\|\kappa\|)$. Following Luxemburg, we call the (external) normed space $\tilde{X}:=(\tilde{X},\|\cdot\|)$ together with the natural embedding

$$
X \stackrel{*}{\rightarrow} \operatorname{Fin}\left({ }^{*} X\right) \stackrel{i}{\rightarrow} \tilde{X}
$$

the norm-nonstandard hull of $X$. In this thesis, we use slightly different name for $\tilde{X}$, which is commonly known as nonstandard hull of $X[1,17,7]$. The reason lies in the fact that the same vector space $X$ may simultaneously carry several different structures together with the norm structure and, hence, $X$ may possess several different types of nonstandard hulls together with the norm-nonstandard hull. There is a well developed theory of norm-nonstandard hulls of normed spaces and operators between them (see, e.g., [17]).

In the case when $X$ is a vector lattice, no norm is available in general. This reason has motivated the study of different kinds of nonstandard hulls of $X$ (see, e.g., [9, 7]). We begin with the following (external) vector spaces over $\mathbb{R}$ associated with the vector lattice $X$ (cf. [7, p.184]). The space

$$
\operatorname{fin}\left({ }^{*} X\right):=\left\{\kappa \in{ }^{*} X:(\exists x \in X)|\kappa| \leq x\right\}
$$

of order-finite elements of ${ }^{*} X$, the space

$$
\lambda\left({ }^{*} X\right):=\left\{\kappa \in{ }^{*} X:(\exists y \in X)(\forall n \in \mathbb{N})|n \kappa| \leq y\right\}
$$


of regular-infinitesimal elements ( $r$-infinitesimals) of ${ }^{*} X$, and the space

$$
\left.\eta\left({ }^{*} X\right):=\left\{\kappa \in{ }^{*} X: \inf _{X}\{x \in X:|\kappa| \leq x\}=0\right\}\right\}
$$

of order-infinitesimal elements (o-infinitesimals) of ${ }^{*} X$.

It is immediate to see that both $\lambda\left({ }^{*} X\right)$ and $\eta\left({ }^{*} X\right)$ are order ideals in the (external) vector lattice $\operatorname{fin}\left({ }^{*} X\right)$ and therefore one may consider the quotients $(r)-X:=$ $\operatorname{fin}\left({ }^{*} X\right) / \lambda\left({ }^{*} X\right)$ and $(o)-X:=\operatorname{fin}\left({ }^{*} X\right) / \eta\left({ }^{*} X\right)$ which are also vector lattices. Following [7, Sect.4.9, 4.8], we call $(r)-X$ the regular-nonstandard hull of $X$ and $(o)-X$ the order-nonstandard hull of $X$.

Notice that the lattice homomorphism

$$
X \stackrel{*}{\rightarrow} \operatorname{fin}\left({ }^{*} X\right) \stackrel{i}{\rightarrow}(o)-X
$$

is always an embedding, while the lattice homomorphism

$$
X \stackrel{*}{\rightarrow} \operatorname{fin}\left({ }^{*} X\right) \stackrel{i}{\rightarrow}(r)-X
$$

is an embedding iff the lattice $X$ is Archimedean. For more information on regular and order-nonstandard hulls of vector lattices we refer to survey [7]. 


\section{CHAPTER 3}

\section{SOME EXTERNAL VECTOR SPACES ASSOCATED WTH AN OVS}

From now on $X=\left(X, X_{+}\right)$is an OVS, unless otherwise stated. As usual, we identify $X$ with its image under the embedding $X \stackrel{*}{\hookrightarrow}{ }^{*} X$. Given $\kappa \in{ }^{*} X$, denote the set of standard upper and lower bounds of $\kappa$ by $U(\kappa):=\{x \in X: \kappa \leq x\}$ and $L(\kappa):=-U(-\kappa)$, respectively. $X$ may not have a lattice structure, but we must choose appropriate analogues of finite/infinitesimal elements to be consistent when $X$ is a vector lattice to boot.

3.1. Consider the following external sets: the set

$$
\operatorname{fin}\left({ }^{*} X\right):=\left\{\kappa \in{ }^{*} X:(\exists x, y \in X) \kappa \in[x, y]\right\}
$$

of order-finite elements of ${ }^{*} X$, the set

$$
\lambda\left({ }^{*} X\right):=\left\{\kappa \in{ }^{*} X:(\exists y \in X)(\forall n \in \mathbb{N}) n \kappa \in[-y, y]\right\}
$$

of regular-infinitesimal elements ( $r$-infinitesimals) of ${ }^{*} X$, the set

$$
\text { r-pns }\left({ }^{*} X\right):=\left\{\kappa \in{ }^{*} X: \bigcap_{n \in \mathbb{N}}(U(n \kappa)-L(n \kappa)) \neq \emptyset\right\}
$$

of regular-pre-near-standard elements of ${ }^{*} X$, the set

$$
\eta\left({ }^{*} X\right):=\left\{\kappa \in{ }^{*} X: \inf _{X} U(\kappa)=\sup _{X} L(\kappa)=0\right\}
$$


of order-infinitesimal elements (o-infinitesimals) of ${ }^{*} X$, and the set

$$
\text { o-pns }\left({ }^{*} X\right):=\left\{\kappa \in{ }^{*} X: \inf _{X}(U(\kappa)-L(\kappa))=0\right\}
$$

of order-pre-near-standard elements of ${ }^{*} X$. The above defined external sets make sense also for an ordered group $X$ but we do not consider this generalization in the present paper. Clearly, the above defined sets are (external) vector spaces over $\mathbb{R}$ satisfying

$$
\begin{gathered}
\eta\left({ }^{*} X\right) \subseteq \text { o-pns }\left({ }^{*} X\right) \subseteq \operatorname{fin}\left({ }^{*} X\right), \lambda\left({ }^{*} X\right) \subseteq \text { r-pns }\left({ }^{*} X\right) \subseteq \text { fin }\left({ }^{*} X\right), \\
X \subseteq \text { r-pns }\left({ }^{*} X\right) \cap \text { o-pns }\left({ }^{*} X\right), \text { and } \eta\left({ }^{*} X\right) \cap X=\{0\} .
\end{gathered}
$$

In general, $X \cap \lambda\left({ }^{*} X\right)$ may contain nonzero elements (for instance, when $X=\left(\mathbb{R}^{2}, \leq\right.$ lex ) ). The inclusions $X+\eta\left({ }^{*} X\right) \subseteq$ o-pns $\left({ }^{*} X\right)$ and $X+\lambda\left({ }^{*} X\right) \subseteq$ r-pns $\left({ }^{*} X\right)$ may be proper (cf. [7, Thm.4.4.2]). If $X$ is Archimedean then $\lambda\left({ }^{*} X\right) \subseteq \eta\left({ }^{*} X\right)$ since $\inf _{n \geq 1} \frac{1}{n} y=0$ for every $y \in X_{+}$. If $X$ is not Archimedean, $\eta\left({ }^{*} X\right)$ may be a proper subset of $\lambda\left({ }^{*} X\right)$ even when $X$ is almost Archimedean and $\operatorname{dim}(X)=2$ (take $X=\left(\mathbb{R}^{2}, \succ\right.$ ) ordered by $(x, y) \succ(0,0)$ if either $x=y=0$ or $x>0$ and $y>0)$.

The sets fin $\left({ }^{*} X\right), \lambda\left({ }^{*} X\right), \mathrm{r}$-pns $\left({ }^{*} X\right), \eta\left({ }^{*} X\right)$, and o-pns $\left({ }^{*} X\right)$ are ordered vector spaces over $\mathbb{R}$ with respect to the ordering inherited from ${ }^{*} X$. Moreover, fin $\left({ }^{*} X\right), \lambda\left({ }^{*} X\right)$, and $\eta\left({ }^{*} X\right)$ are order ideals in ${ }^{*} X, \lambda\left({ }^{*} X\right)$ is an order ideal in r-pns $\left({ }^{*} X\right)$, and $\eta\left({ }^{*} X\right)$ is an order ideal in o-pns $\left({ }^{*} X\right)$.

Proposition 1. Let $X$ be an OVS. Then,

(a) for any $\kappa \in \operatorname{fin}\left({ }^{*} X\right), \kappa \in \lambda\left({ }^{*} X\right)$ iff there exists $v \in{ }^{*} \mathbb{N} \backslash \mathbb{N}$ and $y \in X_{+}$with $v \kappa \in[-y, y]$

(b) the positive cone of $\lambda\left({ }^{*} X\right)$ is generating.

Proof. (a): It follows immediately from the general saturation principle. 
$(b)$ : Let $\kappa \in \lambda\left({ }^{*} X\right)$. Then, by $(a), v \kappa \in[-y, y]$ for some $y \in X_{+}$and $v \in{ }^{*} \mathbb{N} \backslash \mathbb{N}$. Hence

$$
v^{-1} y, \kappa \in\left[-v^{-1} y, v^{-1} y\right] \subset \lambda\left({ }^{*} X\right)
$$

and

$$
v^{-1} y-\kappa \in\left[-2 v^{-1} y, 2 v^{-1} y\right] \subset \lambda\left({ }^{*} X\right)
$$

Therefore,

$$
\kappa=v^{-1} y-\left(v^{-1} y-\kappa\right) \in \lambda\left({ }^{*} X\right)_{+}-\lambda\left({ }^{*} X\right)_{+}
$$

3.2. The following theorem extends [7, Lm.4.6.1.] to OVS.

Theorem 1. Let $X$ be an OVS with the generating positive cone $X_{+}$and let $A$ be $a$ subset of $X_{+}$. Then the following conditions are equivalent:

1) A is order bounded;

2) ${ }^{*} A \subseteq \operatorname{fin}\left({ }^{*} X\right)$

3) ${ }^{*} A \subseteq v \cdot \lambda\left({ }^{*} X\right)$ for every $v \in{ }^{*} \mathbb{N} \backslash \mathbb{N}$;

4) there exists $v \in{ }^{*} \mathbb{N} \backslash \mathbb{N}$ such that ${ }^{*} A \subseteq v \cdot \lambda\left({ }^{*} X\right)$;

5) there exists $v \in{ }^{*} \mathbb{N} \backslash \mathbb{N}$ such that ${ }^{*} A \subseteq v \cdot$ fin $\left({ }^{*} X\right)$.

Moreover, if 1) $\Leftrightarrow 2$ ) in some OVS $X$, then the cone $X_{+}$is generating.

Proof. 1) $\Rightarrow 2$ ): It follows from the transfer principle.

$2) \Rightarrow 1$ ): Since $X_{+}$is generating, we obtain

$$
\operatorname{fin}\left({ }^{*} X\right)=\bigcup_{u \in X_{+}}{ }^{*}[-u, u]
$$


Assume that ${ }^{*} A \subseteq$ fin $\left({ }^{*} X\right)$ but $A$ is not order bounded. The family $\left\{{ }^{*} A \backslash^{*}[-u, u]\right\}_{u \in X_{+}}$ of internal sets possesses the finite intersection property, since

$$
\begin{gathered}
\emptyset \neq *^{*} A \backslash *\left[-\sum_{k=1}^{n} u_{k}, \sum_{k=1}^{n} u_{k}\right] \subseteq \\
{ }^{*} A \backslash \bigcup_{k=1}^{n}{ }^{*}\left[-u_{k}, u_{k}\right]=\bigcap_{k=1}^{n}\left({ }^{*} A \backslash{ }^{*}\left[-u_{k}, u_{k}\right]\right)
\end{gathered}
$$

for every finite subset $\left\{u_{k}\right\}_{k=1}^{n}$ of $X_{+}$. By (1) and the general saturation principle,

$$
{ }^{*} A \backslash \operatorname{fin}\left({ }^{*} X\right)={ }^{*} A \backslash \bigcup_{u \in X_{+}}{ }^{*}[-u, u]=\bigcap_{u \in X_{+}}\left({ }^{*} A \backslash{ }^{*}[-u, u]\right) \neq \emptyset
$$

which violates our hypothesis ${ }^{*} A \subseteq \operatorname{fin}\left({ }^{*} X\right)$. The contradiction shows that $A$ is order bounded.

1) $\Rightarrow 3)$ : Let $A$ be order bounded. Since $X_{+}$is generating, we get $A \subseteq[-u, u]$ for some $u \in X_{+}$. Then $\frac{1}{v}^{*} A \subseteq\left[-\frac{1}{v} u, \frac{1}{v} u\right] \subseteq \lambda\left({ }^{*} X\right)$ for every $v \in{ }^{*} \mathbb{N} \backslash \mathbb{N}$, since if $-\frac{1}{v} u \leq$ $\kappa \leq \frac{1}{v} u$ then

$$
-\frac{1}{n} u \leq-\frac{1}{v} u \leq \kappa \leq \frac{1}{v} u \leq \frac{1}{n} u
$$

for all $n \in \mathbb{N}$.

3) $\Rightarrow 4) \Rightarrow 5$ ): This is obvious.

$5) \Rightarrow 1$ ): Assume that $A$ is not order bounded. By the transfer principle, ${ }^{*} A$ is not order bounded in ${ }^{*} X$. So, $v^{-1 *} A$ is not order bounded in ${ }^{*} X$ as well. Then $\left(v^{-1 *} A\right) \backslash *[-u, u]$ is a nonempty internal set for every $u \in X_{+}$. Arguing as in the proof of implication 2$) \Rightarrow 1$, we obtain that the family $\left\{\left(v^{-1 *} A\right) \backslash{ }^{*}[-u, u]\right\}_{u \in X_{+}}$has the finite intersection property. In view of the general saturating principle,

$$
\left(v^{-1 *} A\right) \backslash \operatorname{fin}\left({ }^{*} X\right)=\left(v^{-1 *} A\right) \backslash \bigcup_{u \in X_{+}} *[-u, u]=\bigcap_{u \in X_{+}}\left(\left(v^{-1 *} A\right) \backslash^{*}[-u, u]\right) \neq \emptyset
$$


that is, ${ }^{*} A \nsubseteq v \cdot \operatorname{fin}\left({ }^{*} X\right)$. The obtained contradiction shows that $A$ is order bounded.

In order to prove the last statement of the Theorem 1, assume that $X_{+}$is nongenerating. Then, there are $x, y \in X$ such that $A=\{x, y\}$ is not order bounded, although ${ }^{*} A=A \subset X \subset \operatorname{fin}\left({ }^{*} X\right)$. Since the implication 1$\left.) \Rightarrow 2\right)$ holds regardless of $X_{+}$being generating or not, we are done.

3.3. In this subsection, we discuss nonstandard interpretations of some additional properties of the external vector spaces defined above. For the order convergence (the $(o)$-convergence, e.g., $[22,28])$ of monotone nets in $X$, there is the following [5, Prop.3] (cf. [7, 4.3.2], [8] for the vector lattice setting) nonstandard characterization:

Proposition 2. For a monotone net $\left(x_{\alpha}\right)_{\alpha \in \Xi}$ in an OVS X, the following conditions are equivalent :

(a) $x_{\alpha} \stackrel{(o)}{\rightarrow} 0$;

(b) $x_{\beta} \in \eta\left({ }^{*} X\right)$ for all $\beta \in{ }^{a} \Xi$;

(c) $x_{\beta} \in \eta\left({ }^{*} X\right)$ for some $\beta \in{ }^{a} \Xi$, where the definition of ${ }^{a} \Xi$ is given in Lemma 1 .

For the $(r)$-convergence, we have [5, Prop.4]:

Proposition 3. For a monotone sequence $\left(x_{n}\right)$ in an OVS $X$, the following conditions are equivalent :
(a) $x_{n} \stackrel{(r)}{\rightarrow} 0$;
(b) $x_{v} \in \lambda\left({ }^{*} X\right)$ for all $v \in{ }^{*} \mathbb{N} \backslash \mathbb{N}$;
(c) $x_{v} \in \lambda\left({ }^{*} X\right)$ for some $v \in{ }^{*} \mathbb{N} \backslash \mathbb{N}$.

The next two results are taken from [5, Lm.1, Thm.2]. 
Lemma 2. Let $X$ be an OVS, $u \in X$, and $v \in{ }^{*} \mathbb{N} \backslash \mathbb{N}$. Then either $u=0$ or $v u \notin$ o-pns $\left({ }^{*} X\right)$.

However, it may be the case that $v u \in \operatorname{fin}\left({ }^{*} X\right)$ for all $v \in{ }^{*} \mathbb{N}$ for some $u \neq 0$ in a non-Archimedean space $X$.

Proposition 4. Any Archimedean OVS X satisfying

o-pns $\left({ }^{*} X\right)=X+\eta\left({ }^{*} X\right)$ is Dedekind complete.

3.4. The following theorem is a refinement of the proposition above.

Theorem 2. Let X be an Archimedean OVS. Consider the following conditions:

1) $X$ is order complete;

2) o-pns $\left({ }^{*} X\right)=X+\eta\left({ }^{*} X\right)$;

3) X is Dedekind complete.

Then 1) $\Rightarrow 2) \Rightarrow 3$ ).

Proof. 1) $\Rightarrow 2)$ : It suffices to show that o-pns $\left({ }^{*} X\right) \subseteq X+\eta\left({ }^{*} X\right)$. Let $\kappa \in$ o-pns $\left({ }^{*} X\right)$. Since $X$ is order complete and $L(\kappa)$ is order bounded in $X$, there exists $a:=\sup _{X} L(\kappa)$. Then $L(\kappa) \leq\{a, \kappa\} \leq U(\kappa)$, and hence

$$
a-\kappa \leq U(\kappa)-L(\kappa)=U(a-\kappa)-L(a-\kappa)
$$

Thus $U(a-\kappa)-L(a-\kappa) \subseteq U(a-\kappa)$. Since $a-\kappa \in$ o-pns $\left({ }^{*} X\right)$, it follows from (2) that $\inf _{X} U(a-\kappa)=0$. A similar argument shows that $\sup _{X} L(a-\kappa)=0$. Hence $a-\kappa \in \eta\left({ }^{*} X\right)$ or $\kappa \in X+\eta\left({ }^{*} X\right)$, as desired.

2) $\Rightarrow 3$ ): It is just Proposition 4 . 
We do not know whether or not 2) implies 1) in Theorem 2. However, if $X$ is Archimedean vector lattice then 2) $\Rightarrow 1$ ) holds by [7, Thm.4.4.2]. Moreover, in this case the quotient space

$$
\text { o-pns }\left({ }^{*} X\right) / \eta\left({ }^{*} X\right) \subseteq(o) X
$$

is a Dedekind completion of $X[7$, Thm.4.4.1].

A short inspection of the proof of [7, Thm.4.4.1] suggests (assuming $X$ to be an Archimedean OVS) that o-pns $\left({ }^{*} X\right) / \eta\left({ }^{*} X\right)$ is a Dedekind completion of $X$ (but not necessarily an order completion).

3.5. The next two theorems give an extended and corrected version of [5, Thm.1] (cf. also [6, Thm.1]). Namely, the equivalent conditions 3)-5) in [6, Thm.1] are supplemented by the last two equivalent conditions of Theorem 4 below.

Theorem 3. Let $X$ be an OVS. Then the following conditions are equivalent:

1) $X$ is almost Archimedean;

2) $\lambda\left({ }^{*} X\right) \cap X=\{0\}$.

Proof. 1) $\Leftrightarrow 2)$ : It follows readily from the definition of $\lambda\left({ }^{*} X\right)$.

Theorem 4. Let $X$ be an OVS. Then the following conditions are equivalent:
1) $X$ is Archimedean;
2) $\lambda\left({ }^{*} X\right) \subseteq \eta\left({ }^{*} X\right)$;
3) $\lambda\left({ }^{*} X\right) \subseteq \mathrm{o}-\mathrm{pns}\left({ }^{*} X\right)$;
4) $\eta\left({ }^{*} X\right)$ is r-closed in $\operatorname{fin}\left({ }^{*} X\right)$;
5) o-pns $\left({ }^{*} X\right)$ is $r$-closed in $\operatorname{fin}\left({ }^{*} X\right)$. 
Proof. 1) $\Rightarrow 2)$ : Let $\kappa \in \lambda\left({ }^{*} X\right)$. Then $-\frac{1}{n} u \leq \kappa \leq \frac{1}{n} u$ for some $u \in X_{+}$and all $n \in$ $\mathbb{N} \backslash\{0\}$. In order to show that $\kappa \in \eta\left({ }^{*} X\right)$, it is sufficient to prove that $\inf _{X} U(\kappa)=0$. Take a $w \in U(\kappa)$. Then $-\frac{1}{n} u \leq \kappa \leq w$ for all $n \in \mathbb{N} \backslash\{0\}$. Since $X$ is Archimedean, $\inf _{n \in \mathbb{N} \backslash\{0\}} \frac{1}{n} u=0$ and

$$
0=-\inf _{n \in \mathbb{N} \backslash\{0\}} \frac{1}{n} u=\sup _{n \in \mathbb{N} \backslash\{0\}}\left(-\frac{1}{n} u\right) \leq w .
$$

We obtain $0 \leq w$, and hence $0 \leq U(\kappa)$. Let $z \in X$ and $z \leq U(\kappa)$. Then $z \leq \frac{1}{n} u$ for all $n \in \mathbb{N} \backslash\{0\}$. As $X$ is Archimedean, we get $z \leq 0$. Thus, $\inf _{X} U(\kappa)=0$, as desired.

2) $\Rightarrow 3)$ : It follows readily from the fact that $\eta\left({ }^{*} X\right) \subseteq$ o-pns $\left({ }^{*} X\right)$.

3) $\Rightarrow 1$ ): It suffices to show that

$$
\left[(\forall n \in \mathbb{N}) n y \leq u \in X_{+}\right] \Rightarrow[y \leq 0]
$$

Take a $y \in X$, so that $n y \leq u \in X_{+}$for all $n \in \mathbb{N}$. Fix $v \in{ }^{*} \mathbb{N} \backslash \mathbb{N}$. Then $\frac{1}{v} u \in \lambda\left({ }^{*} X\right) \subseteq$ o-pns $\left({ }^{*} X\right)$. Let $z \in X$, then, by the transfer principle, $\frac{1}{v} u \leq z$ iff $\frac{1}{n} u \leq z$ for some $n \in \mathbb{N} \backslash\{0\}$. Therefore,

$$
U\left(\frac{1}{v} u\right)=\bigcup_{n \in \mathbb{N} \backslash\{0\}} U_{n}, \text { where } U_{n}=U\left(\frac{1}{n} u\right) .
$$

By the hypothesis, $\inf _{X}\left(U\left(\frac{1}{v} u\right)-L\left(\frac{1}{v} u\right)\right)=0$. Since $0 \in L\left(\frac{1}{v} u\right)$, then $\inf _{X} U\left(\frac{1}{v} u\right)=$ $\inf _{X}\left(U\left(\frac{1}{v} u\right)-0\right)=0$. Thus

$$
\inf _{X} \bigcup_{n \in \mathbb{N} \backslash\{0\}} U_{n}=0 .
$$

Since $y \leq \frac{1}{n} u \in U_{n}$ for all $n \in \mathbb{N} \backslash\{0\}$, then it follows from (3), that $y \leq 0$, as desired.

$1) \Rightarrow 4)$ : Take a sequence $\left(\kappa_{n}\right)_{n}$ in $\eta\left({ }^{*} X\right)$ which $r$-converges to $\kappa \in \operatorname{fin}\left({ }^{*} X\right)$. It is enough to show that $\kappa \in \eta\left({ }^{*} X\right)$. We may suppose that $\kappa_{n} \stackrel{u}{\rightarrow} \kappa$ for some $u \in X_{+}$. So, 
there is a sequence $\varepsilon_{n} \downarrow 0$ of reals such that $-\varepsilon_{n} u \leq \kappa_{k}-\kappa \leq \varepsilon_{n} u$ for all naturals $k \geq n$. Then, $\kappa_{n}-\varepsilon_{n} u \leq \kappa \leq \kappa_{n}+\varepsilon_{n} u$, and therefore, $L\left(\kappa_{n}\right)-\varepsilon_{n} u \subseteq L(\kappa)$, and $U\left(\kappa_{n}\right)+\varepsilon_{n} u \subseteq$ $U(\kappa)$ for every $n \in \mathbb{N}$. In view of $\left(\kappa_{n}\right)_{n} \subseteq \eta\left({ }^{*} X\right)$, one gets

$$
-\varepsilon_{n} u \leq \kappa \leq \varepsilon_{n} u \quad(\forall n \in \mathbb{N})
$$

Since $X$ is Archimedean, $u \in X_{+}$, and $\varepsilon_{n} \downarrow 0$, (4) implies that $\inf _{X} U(\kappa)=0=\sup _{X} L(\kappa)$. Thus, $\kappa \in \eta\left({ }^{*} X\right)$, as desired.

$4) \Rightarrow 1$ ): Suppose that $X$ is not Archimedean. Then, there are vectors $u \in X_{+}$ and $v \in X \backslash\left(-X_{+}\right)$such that $n v \leq u$ for all $n \in \mathbb{N}$. Take some $v \in * \mathbb{N} \backslash \mathbb{N}$. Clearly, $\frac{1}{v} u \notin \eta\left({ }^{*} X\right)$ since $\inf _{X} U\left(\frac{1}{v} u\right)$ does not exist. However, the sequence $\left(x_{n}\right)_{n \in \mathbb{N}}$, where $x_{n}=0 \in \eta\left({ }^{*} X\right)$ for all $n \in \mathbb{N}$, converges $u$-uniformly to $\frac{1}{v} u$. By the hypothesis of the $r$-closeness of $\eta\left({ }^{*} X\right), \frac{1}{v} u \in \eta\left({ }^{*} X\right)$. The obtained contradiction shows that $X$ is Archimedean.

1) $\Rightarrow 5)$ : Take a sequence $\left(\kappa_{n}\right)_{n}$ in o-pns $\left({ }^{*} X\right)$ which $r$-converges to $\kappa \in \operatorname{fin}\left({ }^{*} X\right)$. It is enough to show that $\kappa \in$ o-pns $\left({ }^{*} X\right)$. One may suppose that $\kappa_{n} \stackrel{u}{\rightarrow} \kappa$ for some $u \in X_{+}$. So, there is a sequence $\varepsilon_{n} \downarrow 0$ of reals such that $-\varepsilon_{n} u \leq \kappa_{k}-\kappa \leq \varepsilon_{n} u$ for all naturals $k \geq n$. For every $n \in \mathbb{N}$, we have $\kappa_{n}-\varepsilon_{n} u \leq \kappa \leq \kappa_{n}+\varepsilon_{n} u$, and hence

$$
L\left(\kappa_{n}-\varepsilon_{n} u\right) \leq \kappa \leq U\left(\kappa_{n}+\varepsilon_{n} u\right) .
$$

Given $n \in \mathbb{N}$, assign $\mathscr{H}_{n}:=U\left(\kappa_{n}+\varepsilon_{n} u\right)-L\left(\kappa_{n}-\varepsilon_{n} u\right)$. The inclusion $\left(\kappa_{n}\right)_{n} \subseteq$ o-pns $\left({ }^{*} X\right)$ implies, by (5), that

$$
\inf _{X} \mathscr{H}_{n}=2 \varepsilon_{n} u \quad(\forall n \in \mathbb{N}) .
$$

Since the OVS $X$ is Archimedean, (6) implies that $\inf _{X} \bigcup_{n=1}^{\infty} \mathscr{H}_{n}=0$ Therefore, $\inf _{X}(U(\kappa)-L(\kappa))=0$ (using the inclusion $\bigcup_{n=1}^{\infty} \mathscr{H}_{n} \subseteq$ $U(\kappa)-L(\kappa)$, which is ensured by (5)). Thus, $\kappa \in \mathrm{o}-\mathrm{pns}\left({ }^{*} X\right)$, as required. 
5) $\Rightarrow 1)$ : Suppose that $X$ is not Archimedean. Then, there are $u \in X_{+}, v \in X \backslash$ $\left(-X_{+}\right)$with $n v \leq u$ for all $n \in \mathbb{N}$. Take a $v \in * \mathbb{N} \backslash \mathbb{N}$. Since $\inf _{X}\left(U\left(\frac{1}{v} u\right)-L\left(\frac{1}{v} u\right)\right)$ does not exist, we get $\frac{1}{v} u \notin \mathrm{o}-\operatorname{pns}\left({ }^{*} X\right)$. The sequence $\left(x_{n}\right)_{n \in \mathbb{N}}$ with $x_{n}=0 \in \eta\left({ }^{*} X\right)$ for all $n \in \mathbb{N}$ converges $u$-uniformly to $\frac{1}{v} u$. But, by the hypothesis of the uniform closeness of o-pns $\left({ }^{*} X\right)$, we get $\frac{1}{v} u \in \eta\left({ }^{*} X\right)$. The obtained contradiction shows that $X$ is Archimedean. 


\section{CHAPTER 4}

\section{THE ORDER AND THE REGULAR NONSTANDARD HULLS OF AN OVS}

4.1. Let $X$ be an OVS and consider the following quotient OVS equipped with the canonical partial ordering:

$$
{ }_{(o)} X:=\operatorname{fin}\left({ }^{*} X\right) / \eta\left({ }^{*} X\right)
$$

The mapping $X \stackrel{\hat{\eta}}{\rightarrow}_{(o)} X$ defined by $\hat{\eta}(x)=\left[{ }^{*} x\right]$ is an order embedding since $\eta\left({ }^{*} X\right) \cap X=$ $\{0\}$ OVS $_{(o)} X$ is called the order (nonstandard) hull of $X$.

It is known that the order hull of an Archimedean vector lattice is Archimedean [7, Thm.4.8.3]. The reason lies in $r$-closeness of $\eta\left({ }^{*} X\right)$ by Theorem 4 and in the fact that the quotient of any vector lattice by an $r$-closed order ideal is Archimedean by Veksler's theorem (cf. [27], [22, Thm.60.2]).

In general, ${ }_{(o)} X$ is not $o$-complete even if $X$ is a Dedekind complete vector lattice, for example $L_{p}[0,1]$ with $0<p<\infty($ see $[7$, p.202])

4.2. Consider the following quotient space with respect to the canonical partial ordering:

$$
(r) X:=\operatorname{fin}\left({ }^{*} X\right) / \lambda\left({ }^{*} X\right)
$$

The corresponding order homomorphism $X \stackrel{\hat{\lambda}}{\rightarrow}{ }_{(r)} X, \lambda(x)=\left[{ }^{*} x\right]$, is an order embedding iff $\lambda\left({ }^{*} X\right) \cap X=\{0\}$, and, by Theorem 4, iff $X$ is almost Archimedean. For an almost Archimedean OVS $X$, the OVS ${ }_{(r)} X$ is called the regular (nonstandard) hull of $X$. 
According to [7, Cor.4.9.6], the regular hull of any Banach lattice is Archimedean. For an arbitrary almost Archimedean OVS $X$, it is still unknown whether or not ${ }_{(r)} X$ is almost Archimedean, even if $X$ is a vector lattice [7, p.214]. The challenge here may pertain to establishment of the $r$-closeness of $\lambda\left({ }^{*} X\right)$ even in the case if $X$ is a vector lattice.

If $X=S A(H)$ the algebra of all selfadjoint linear operators on a Hilbert space $H$, then $X$ is a Krein spaces with the normal cone of all positive operators and by Corollary $1, \tilde{X}={ }_{(r)} X$. Since $\tilde{X}$ is a normed lattice, it is Archimedean and, therefore, ${ }_{(r)} X$ is also Archimedean.

Theorem 5. Let $X$ be an OVS, in which for every sequence $z_{k} \in X$, there exists a sequence of reals $\alpha_{k}>0$ such that the set $\left\{\alpha_{k} z_{k}: k \in \mathbb{N}\right\}$ is order bounded above. Then ${ }_{(r)} X$ is almost Archimedean.

Proof. Note that it follows immediately from the assumption of the theorem that $X_{+}$ is generating. Let $[\kappa],[\zeta] \in{ }_{(r)} X$ satisfy

$$
\pm[\kappa] \leq n^{-1}[\zeta] \quad(\forall n \in \mathbb{N}) .
$$

Take $y \in X$ so that $\zeta \leq y$ then

$$
\pm[\kappa] \leq n^{-1}[y] \quad(\forall n \in \mathbb{N})
$$

Therefore, there exist two sequences $\xi_{n}^{\prime}, \xi_{n}^{\prime \prime} \in \lambda\left({ }^{*} X\right)$ such that

$$
\kappa \leq n^{-1} y+\xi_{n}^{\prime}, \quad-\kappa \leq n^{-1} y+\xi_{n}^{\prime \prime} \quad(\forall n \in \mathbb{N})
$$

Since $\lambda\left({ }^{*} X\right)_{+}-\lambda\left({ }^{*} X\right)_{+}=\lambda\left({ }^{*} X\right)$ by Proposition 1 , there exists $\xi_{n} \in \lambda\left({ }^{*} X\right)_{+}$such that $\xi_{n} \geq \xi_{n}^{\prime}, \xi_{n}^{\prime \prime}$ and hence

$$
\pm \kappa \leq n^{-1} y+\xi_{n} \quad(\forall n \in \mathbb{N}) .
$$


Since $\xi_{n} \in \lambda\left({ }^{*} X\right)_{+}$, there is a sequence $z_{n} \in X_{+}$satisfying

$$
0 \leq \xi_{n} \leq k^{-2} z_{n} \quad(\forall k, n \in \mathbb{N})
$$

By the general saturation principle, there is $v \in{ }^{*} \mathbb{N} \backslash \mathbb{N}$ with

$$
0 \leq \xi_{n} \leq v^{-2} z_{n} \quad(\forall n \in \mathbb{N})
$$

By the assumption of the theorem, there is $z \in X_{+}$such that

$$
0 \leq \alpha_{n} z_{n} \leq z \quad\left(\alpha_{n}>0, n \in \mathbb{N}\right)
$$

Then

$$
0 \leq v^{-2} z_{n} \leq v^{-1} \alpha_{n} z_{n} \leq v^{-1} z \quad(\forall n \in \mathbb{N})
$$

It follows from (8) and (9) that $0 \leq \xi_{n} \leq v^{-1} z$, and hence, by (7)

$$
\pm \kappa \leq n^{-1} y+v^{-1} z \quad(\forall n \in \mathbb{N}) .
$$

Then

$$
\pm \kappa \leq n^{-1}(y+z) \quad(\forall n \in \mathbb{N}) .
$$

which means that $\kappa \in \lambda\left({ }^{*} X\right)$ and therefore, $[\kappa]=0$. We have shown that ${ }_{(r)} X$ is almost Archimedean

Theorem 6. Let $X$ be a Banach OVS with the closed and generating cone $X_{+}$. Then ${ }_{(r)} X$ is Archimedean.

Proof. It suffices to check if the hypothesis of Theorem 5 is satisfied. Let $z_{k} \in X$. Since $X_{+}$is generating, there exists a sequence $y_{k} \in X_{+}$with $z_{k} \leq y_{k}$. Since $X$ is a Banach 
space and the cone $X_{+}$is closed, $y:=\sum_{n=1}^{\infty} \frac{y_{n}}{2^{n}\left\|y_{n}\right\|} \in X_{+}$. Taking the norm limits in the inequality

$$
\frac{1}{2^{k}\left\|y_{k}\right\|} z_{k} \leq \frac{1}{2^{k}\left\|y_{k}\right\|} y_{k} \leq \sum_{n=1}^{k+m} \frac{y_{n}}{2^{n}\left\|y_{n}\right\|}
$$

as $m \rightarrow \infty$ and using closeness of $X_{+}$, we obtain

$$
\frac{1}{2^{k}\left\|y_{k}\right\|} z_{k} \leq y \quad(\forall k \in \mathbb{N})
$$

as required. 


\section{CHAPTER 5}

\section{NORMED OVS}

5.1. In this section, $\left(X, X_{+},\|\cdot\|\right)$ is a normed OVS. If $e \in X$ is an order unit, one may consider also the following seminorm on $X$

$$
\|x\|_{e}:=\inf \left\{\lambda \in \mathbb{R}_{+}:-\lambda e \leqslant x \leqslant \lambda e\right\}
$$

which is also a norm when $X$ is Archimedean.

Theorem 7. Let $(X,\|\cdot\|)$ be a normed OVS with a normal generating cone $X_{+}$. Then the following conditions are equivalent:

1) $X$ possesses an order unit e, and the norm $\|\cdot\|_{e}$ is equivalent to $\|\cdot\|$;

2) $\operatorname{Fin}\left({ }^{*} X\right)=\operatorname{fin}\left({ }^{*} X\right)$;

3) $\mu\left({ }^{*} X\right) \subseteq$ fin $\left({ }^{*} X\right)$;

4) $\mu\left({ }^{*} X\right)=\lambda\left({ }^{*} X\right)$;

5) $\mu\left({ }^{*} X\right) \subseteq \lambda\left({ }^{*} X\right)$;

6) $\operatorname{Fin}\left({ }^{*} X\right)=\operatorname{fin}\left({ }^{*} X\right)+\mu\left({ }^{*} X\right)$.

Proof. Since both Fin $\left({ }^{*} X\right)$ and $\mu\left({ }^{*} X\right)$ do not depend on choice of an equivalent norm in $X$ and the cone $X_{+}$is normal, we may and do assume that $\|\cdot\|$ is monotone.

First, we prove the equivalence 2) $\Leftrightarrow 3) \Leftrightarrow 4) \Leftrightarrow 5$ ).

Since the implications 2) $\Rightarrow 3$ ) and 4) $\Rightarrow 5) \Rightarrow 3$ ) do not require checking, it is enough to show that 3$) \Rightarrow 4$ ) and 4$) \Rightarrow 2$ ). 
$3) \Rightarrow 4)$ : Take an arbitrary $\kappa \in \mu\left({ }^{*} X\right)$. Then $v \kappa \in \mu\left({ }^{*} X\right)$ for some $v \in{ }^{*} \mathbb{N} \backslash \mathbb{N}$. Thus, by hypothesis, $v \kappa \in \operatorname{fin}\left({ }^{*} X\right)$. Hence $\kappa \in \lambda\left({ }^{*} X\right)$. So, $\mu\left({ }^{*} X\right) \subseteq \lambda\left({ }^{*} X\right)$. The reverse inclusion $\lambda\left({ }^{*} X\right) \subseteq \mu\left({ }^{*} X\right)$ is trivial.

$4) \Rightarrow 2)$ : Let $\mu\left({ }^{*} X\right)=\lambda\left({ }^{*} X\right)$. By normality of $X_{+}$, fin $\left({ }^{*} X\right) \subseteq \operatorname{Fin}\left({ }^{*} X\right)$. Assume that the inclusion is proper. Then, there exists $\kappa \in{ }^{*} X$ such that $\|\kappa\|=1$ and $\kappa \notin \operatorname{fin}\left({ }^{*} X\right)$ and, in particular, $\kappa \notin[-y, y]$ for all $y \in X_{+}$. Consider the internal sets

$$
A_{y}^{n}:=\left\{r \in{ }^{*} \mathbb{R}_{+}: n \leqslant r, \kappa \notin[-r y, r y]\right\} \quad\left(y \in X_{+}, n \in \mathbb{N}\right) .
$$

Since $\kappa \notin[-n y, n y]$ for every $y \in X_{+}$and $n \in \mathbb{N}$, we have $n \in A_{y}^{n}$, that is, all of these sets are nonempty. The family $\left\{A_{y}^{n}: y \in X_{+}, n \in \mathbb{N}\right\}$ has the finite intersection property, since

$$
A_{y+z}^{\max (n, m)} \subseteq A_{y}^{n} \cap A_{z}^{m}
$$

Thus, by the general saturation principle, there exist $r \in{ }^{*} \mathbb{R}$ satisfying

$$
r \in \bigcap\left\{A_{y}^{n}: y \in X_{+}, n \in \mathbb{N}\right\}
$$

Then $r$ is an infinite positive number such that $\kappa \notin[-r y, r y]$ for all $y \in X_{+}$. However, $(1 / r) \kappa \in \mu\left({ }^{*} X\right)$, since $\|(1 / r) \kappa\|=1 / r \approx 0$. By the hypothesis, $\mu\left({ }^{*} X\right)=\lambda\left({ }^{*} X\right) \subset$ fin $\left({ }^{*} X\right)$, that is. there exists $z \in X_{+}$, such that $(1 / r) \kappa \in[-z, z]$ or $-r z \leqslant \kappa \leqslant r z$, which contradicts the fact that $r \in A_{z}^{1}$.

So 4$) \Rightarrow 2$ ) and the equivalence 2) $\Leftrightarrow 5)$ are established.

Since 1$) \Rightarrow 2) \Rightarrow 6$ ) is trivial, it suffices to prove 6$) \Rightarrow 1$ ).

$6) \Rightarrow 1)$ : Suppose that $\operatorname{Fin}\left({ }^{*} X\right)=\operatorname{fin}\left({ }^{*} X\right)+\mu\left({ }^{*} X\right)$. First, we prove that the unit ball $B:=\{x \in X:\|x\| \leqslant 1\}$ is order bounded. Assume the contrary. Take an arbitrary nonzero $x \in X_{+}$. Then, there exists $y \in X_{+}$with $\|y\|=1$ and $y \notin[0, x]$, for otherwise 
$B$ will be order bounded. Let $z \in\left[\frac{x}{2}+y, x+y\right]$. Then, we have $y<\frac{x}{2}+y \leq z \leq x+y$. Thus,

$$
1=\|y\|<\|z\| \leqslant\|x+y\| \leqslant\|x\|+\|y\|=\|x\|+1 .
$$

Also, since $z-\frac{x}{2} \geqslant y \geqslant 0$, we have $\left\|z-\frac{x}{2}\right\| \geqslant\|y\|=1$. Consequently, the internal sets

$$
A_{x}:=\left\{z \in{ }^{*} X:\left\|z-\frac{x}{2}\right\| \geq 1\right\}
$$

are nonempty for all $x \in X_{+} \backslash\{0\}$. Since $A_{x+y} \subseteq A_{x} \cap A_{y}$ for all $x, y \in X_{+} \backslash\{0\}$, the family $\left\{A_{x}\right\}_{x \in X_{+} \backslash\{0\}}$ satisfies the finite intersection property. Thus, by the general saturation principle, there exists $z_{0} \in{ }^{*} X \backslash\{0\}$ such that

$$
z_{0} \in \bigcap\left\{A_{x}: x \in X_{+} \backslash\{0\}\right\}
$$

Clearly, $z_{0} \in \operatorname{Fin}\left({ }^{*} X\right)$. By hypothesis, $z_{0} \in \operatorname{fin}\left({ }^{*} X\right)+\mu\left({ }^{*} X\right)$. Therefore, there are elements $x_{0} \in X_{+}$and $h \in \mu\left({ }^{*} X\right)$, for which, $z_{0} \leqslant x_{0}+h$. We may suppose $x_{0}>0$, for otherwise, $z_{0} \leq h$ would yield $\left\|z_{0}\right\| \leqslant\|h\| \approx 0$ contradicting the fact that $\left\|z_{0}\right\|>1$. But, if $x_{0}>0$, then $z_{0} \in A_{2 x_{0}}$, that is, $\left\|z_{0}-x_{0}\right\| \geqslant 1$. The obtained contradiction shows that $B$ is order bounded.

Choose an $e$ such that $-e \leqslant y \leqslant e$ for all $y \in B$. Take an arbitrary nonzero $x \in X$. Then, $\frac{x}{\|x\|} \in B$, that is $-e \leqslant \frac{x}{\|x\|} \leqslant e$. Thus, $-\|x\| e \leqslant x \leqslant\|x\| e$. Therefore, $e$ is an order unit.

Recall that $\|x\|_{e}=\inf \{\lambda \in \mathbb{R}:-\lambda e \leqslant x \leqslant \lambda e\}$. Thus $\|x\| \leqslant\|x\|_{e} \cdot\|e\|$. Also, $\|x\|_{e} \leqslant\|x\|$. Consequently, the norms $\|\cdot\|_{e}$ and $\|\cdot\|$ are equivalent. The implication 6) $\Rightarrow 1$ ) is established. The proof of the theorem is complete.

Note that the normality condition for $X_{+}$cannot be dropped in Theorem 7. Indeed, in the normed OVS $X=C^{1}[0,1]$ of differentiable functions on the interval $[0,1]$ we have $\mu\left({ }^{*} X\right) \subseteq \operatorname{fin}\left({ }^{*} X\right)$, however $\operatorname{Fin}\left({ }^{*} X\right)$ is a proper subset of $\operatorname{fin}\left({ }^{*} X\right)$. 
Theorem 8. The norm $\|\cdot\|$ in a normed OVS $(X,\|\cdot\|)$ with a normal cone is order continuous if and only if $\eta\left({ }^{*} X\right) \subseteq \mu\left({ }^{*} X\right)$.

Proof. Assume that the norm $\|\cdot\|$ is order continuous. Take an arbitrary $\kappa \in \eta\left({ }^{*} X\right)$ and assume that $\kappa \notin \mu\left({ }^{*} X\right)$, that is, $\|\kappa\| \not \approx$. Then, there exists $n_{0} \in \mathbb{N}$ such that $\|\kappa\| \geq \frac{1}{n_{0}}$. WLOG suppose that $\kappa>0$. Define $A_{n}:=\left\{x \in X_{+}:\|x\| \geq \frac{1}{n}\right\}$ for each $n \in \mathbb{N}$. Note that, since the norm $\|\cdot\|$ is order continuous, $A_{n}$ is closed in $\mathrm{X}$ with respect to both norm and order topology (actually, they coincide, since the cone is normal) for all $n \in \mathbb{N}$. Also, $0 \notin A_{n}$ for each $n \in \mathbb{N}$. Note that $U(\kappa) \subseteq A_{n_{0}}$ for some $n_{0} \in \mathbb{N}$, that is, $\inf _{X} U(\kappa)>0$, if it exists. Nevertheless, a contradiction to the fact that $\kappa \in \eta\left({ }^{*} X\right)$. Therefore, $\kappa \in \mu\left({ }^{*} X\right)$, or in other words, $\eta\left({ }^{*} X\right) \subseteq \mu\left({ }^{*} X\right)$.

Now, let $\eta\left({ }^{*} X\right) \subseteq \mu\left({ }^{*} X\right)$, and assume that $\rho$ is not order continuous. Thus, there are a net $\left(x_{\xi}\right)_{\xi \in \Theta} \subseteq X, x_{\xi} \downarrow 0$, and a number $0<b \in \mathbb{R}$

such that $\left\|x_{\xi}\right\| \geq b$ for all $\xi \in \Theta$. Take some infinitely remote element $\beta \in^{a} \Theta$. Then, by Proposition 2 , since $x_{\xi} \downarrow 0, x_{\beta} \in \eta\left({ }^{*} X\right)$. Thus, $\left\|x_{\beta}\right\| \approx 0$. On the other hand, by the transfer principle, $\left\|x_{\xi}\right\| \geq b$ for all $\xi \in^{*} \Theta$. The contradiction shows that the norm $\|\cdot\|$ is order continuous.

5.2. Given a normed space $X$, choose a hyperfinite dimensional internal subspace $V$ of ${ }^{*} X$ such that $X \subset V \subset{ }^{*} X$. By the transfer principle, $V$ is internally reflexive, that is: for every $\phi \in V^{\prime \prime}$ there is a unique $\kappa_{\phi} \in V$ with

$$
\phi(\psi)=\psi\left(\kappa_{\phi}\right) \quad\left(\forall \psi \in V^{\prime}\right)
$$

So, we get the norm preserving linear embedding

$$
g \rightarrow\left[\kappa_{g}\right] \quad\left(g \in X^{\prime \prime}\right)
$$


of $X^{\prime \prime}$ into $\tilde{X}$. It is easy to show that this embedding does not depend on our choice of $V$ (the same construction is applicable, due to the general saturation principle, to the diagram $X \hookrightarrow X^{\prime \prime} \hookrightarrow \ldots \hookrightarrow X^{(2 n)} \hookrightarrow \ldots$, namely, every $X^{(2 n)}$ can be embedded naturally into $\tilde{X})$.

Now we have the following preliminary characterization of cone normality.

Theorem 9. Let X be a normed OVS. Then the following conditions are equivalent:

1) the cone $X_{+}$is normal;

2) $\mu\left({ }^{*} X\right)$ is an order ideal in the nonstandard extension $\left({ }^{*} X,{ }^{*}\left(X_{+}\right)\right)$of the OVS $\left(X, X_{+}\right)$;

3) the set

$$
K_{X}=\left\{[\kappa]: \kappa \in{ }^{*}\left(X_{+}\right),\|\kappa\| \in \operatorname{Fin}\left({ }^{*} \mathbb{R}\right)\right\}
$$

is a norm closed cone in $\tilde{X}$;

4) $K_{X}$ is a cone which is normal in $\tilde{X}$.

Proof. 1) $\Rightarrow 2$ ): It follows from the fact that every order interval is norm bounded due to normality of $X_{+}$.

$2) \Rightarrow 3)$ : If $\mu\left({ }^{*} X\right)$ is an order ideal in $\left({ }^{*} X,{ }^{*}\left(X_{+}\right)\right)$, then $\mu\left({ }^{*} X\right)$ is an order ideal in $\left(\operatorname{Fin}\left({ }^{*} X\right),{ }^{*}\left(X_{+}\right) \cap \operatorname{Fin}\left({ }^{*} X\right)\right)$. Hence, the norm-nonstandard hull $\tilde{X}=\operatorname{Fin}\left({ }^{*} X\right) / \mu\left({ }^{*} X\right)$ is a normed OVS with the positive cone

$$
K_{X}=\left\{[\kappa]: \kappa \in^{*}\left(X_{+}\right) \cap \operatorname{Fin}\left({ }^{*} X\right)\right\} .
$$

Clearly, $K_{X}$ is norm closed.

$3) \Rightarrow 4)$ : Assume that $K_{X}$ is not a normal cone in $\tilde{X}$. Then for every $n \in \mathbb{N}$ there are $\left[\kappa_{1}^{n}\right],\left[\kappa_{2}^{n}\right] \in K_{X}$ with

$$
\left\|\left[\kappa_{1}^{n}\right]\right\|=\left\|\left[\kappa_{2}^{n}\right]\right\|=1 \text { and }\left\|\left[\kappa_{1}^{n}\right]+\left[\kappa_{2}^{n}\right]\right\|<1 / n \text {. }
$$


Since $\operatorname{st}(\|\kappa\|)=\|[\kappa]\|$ for every $\kappa \in \operatorname{Fin}\left({ }^{*} X\right)$, one gets

$$
1 / 2 \leq\left\|\kappa_{1}^{n}\right\| \text { and } 1 / 2 \leq\left\|\kappa_{2}^{n}\right\| \text { and }\left\|\kappa_{1}^{n}+\kappa_{2}^{n}\right\|<1 / n
$$

for all $n \in \mathbb{N}$. By the general saturation principle, there exist $v \in{ }^{*} \mathbb{N} \backslash \mathbb{N}$ and two elements $\kappa_{1}^{v}, \kappa_{2}^{v} \in{ }^{*}\left(X_{+}\right)$such that

$$
\left\|\kappa_{1}^{v}\right\|=\left\|\kappa_{2}^{v}\right\|=1 \text { and }\left\|\kappa_{1}^{v}+\kappa_{2}^{v}\right\|<1 / v .
$$

Therefore, $0 \neq\left[\kappa_{1}^{\nu}\right] \in K_{X}$ and $0 \neq\left[\kappa_{2}^{\nu}\right] \in K_{X}$. On the other hand, $\operatorname{st}\left(\left\|\kappa_{1}^{\nu}+\kappa_{2}^{v}\right\|\right)=0$ implies that $\left[\kappa_{2}^{V}\right]=-\left[\kappa_{1}^{V}\right]$ violating the assumption that $K_{X}$ is a cone. The obtained contradiction shows that $K_{X}$ is a normal cone in $\tilde{X}$.

$4) \Rightarrow 3)$ : It is trivial since $\left(X, X_{+}\right)$is an ordered subspace in $\left(\tilde{X}, K_{X}\right)$.

Now we are in the position to prove the following theorem.

Theorem 10. Let $X$ be a Krein space. Then the following conditions are equivalent:

1) the cone $X_{+}$is normal;

2) $\operatorname{Fin}\left({ }^{*} X\right)=\operatorname{fin}\left({ }^{*} X\right)$;

3) $\mu\left({ }^{*} X\right)=\lambda\left({ }^{*} X\right)$;

4) $\operatorname{Fin}\left({ }^{*} X\right)=\operatorname{fin}\left({ }^{*} X\right)+\mu\left({ }^{*} X\right)$.

Proof. Note that the cone $X_{+}$is generating.

1) $\Rightarrow 2$ ): Since in the Krein space $X$ with the normal positive cone $X_{+}$the norms $\|\cdot\|$ and $\|\cdot\|_{u}$ are equivalent, it follows from Theorem 7 that $\operatorname{Fin}\left({ }^{*} X\right)=\operatorname{fin}\left({ }^{*} X\right)$.

2) $\Rightarrow 3):$ If $\kappa \in \mu\left({ }^{*} X\right)$ then

$$
n \kappa \in \mu\left({ }^{*} X\right) \subset \operatorname{Fin}\left({ }^{*} X\right)=\operatorname{fin}\left({ }^{*} X\right) \quad(\forall n \in \mathbb{N}) .
$$


Thus, there exist $a, b \in X$ with $n \kappa \in[a, b]$ for all $n \in \mathbb{N}$. Since $X_{+}$is generating, we may assume that $a=-b$. By the general saturation principle, there exists a hyperfinite natural $v \in{ }^{*} \mathbb{N} \backslash \mathbb{N}$ with $v \kappa \in[-b, b]$. Hence

$$
\kappa \in \frac{1}{v}[-b, b] \subset \lambda\left({ }^{*} X\right) .
$$

If $\kappa \in \lambda\left({ }^{*} X\right)$ then

$$
n \kappa \in \lambda\left({ }^{*} X\right) \subset \operatorname{fin}\left({ }^{*} X\right)=\operatorname{Fin}\left({ }^{*} X\right) \quad(\forall n \in \mathbb{N}) .
$$

Thus $\operatorname{st}(\|\kappa\|)=0$ and hence $\kappa \in \mu\left({ }^{*} X\right)$.

$3) \Rightarrow 2$ ): The proof is based on the same arguments in the reverse direction, as the proof of 2) $\Rightarrow 3$ ) and therefore is omitted.

$2) \Leftrightarrow 4)$ : It is obvious.

$2) \Rightarrow 1)$ : By the condition 2$), \mu\left({ }^{*} X\right)=\lambda\left({ }^{*} X\right)$ is an order ideal in $\operatorname{fin}\left({ }^{*} X\right)=\operatorname{Fin}\left({ }^{*} X\right)$. Then $\mu\left({ }^{*} X\right)$ is an order ideal in ${ }^{*} X$. It follows from Theorem 9 that the cone $X_{+}$is normal in $X$, as required

Notice that without an order unit in $X$, the conditions 1) and 2) of Theorem 10 are not equivalent. For example, in the Banach OVS $\ell^{1}$ of absolutely summable real sequences, the positive cone $\ell_{+}^{1}$ is normal and $e_{v} \in \operatorname{Fin}\left({ }^{*} \ell^{1}\right) \backslash$ fin $\left({ }^{*} \ell^{1}\right)$ for any $v \in{ }^{*} \mathbb{N} \backslash$ $\mathbb{N}$, where $e_{v}$ is the internal sequence with all zero coordinates except $v$-th coordinate which is 1 .

Corollary 1. For any Krein space $X$ with the normal positive cone, its norm-nonstandard hull $\tilde{X}$ coincides with the regular hull ${ }_{(r)} X$.

Again as above, ${ }_{(r)} \ell^{1} \cong \ell^{1}$ but $\tilde{\ell}^{1}$ is much bigger than $\ell^{1}$. 
5.3. Another consequence of Theorem 9 is the following result.

Theorem 11. Let $X$ be a Banach OVS with closed and normal positive cone $X_{+}$. Then the following conditions are equivalent:

1) $\tilde{X}$ has the Levi property;

2) $\tilde{X}$ is Dedekind complete;

3) $\tilde{X}$ is $\sigma$-Dedekind complete.

Proof. By Theorem 9, $\tilde{X}$ is an ordered Banach space with closed and normal positive cone $K_{X}$.

1) $\Rightarrow 2) \Rightarrow 3)$ : This is true in any ordered Banach space.

$3) \Rightarrow 1)$ : Let $0 \leq\left[\kappa_{n}\right] \uparrow \leq[\xi]$ in $\tilde{X}$. Then there is $\left[\kappa_{0}\right]=\sup _{\tilde{X}}\left\{\left[\kappa_{n}\right]: n \in \mathbb{N}\right\}$. It is easy to construct a sequence $\left(\kappa_{n}^{1}\right)_{n \in \mathbb{N}}$ in ${ }^{*} X$ by induction, such that

$$
0 \leq \kappa_{n}^{1} \uparrow \leq \kappa_{0}^{1} \text { and } \operatorname{st}\left(\left\|\kappa_{n}^{1}-\kappa_{n}\right\|\right)=0
$$

for all $n=1,2, \ldots$. It is enough to show that $\left(\left[\kappa_{n}^{1}\right]\right)_{n \in \mathbb{N}}$ is a Cauchy sequence. Assume that it is not Cauchy. Then, without lost of generality. we may suppose that $\| \kappa_{n+1}^{1}-$ $\kappa_{n}^{1} \|=1$ and $\left\|\kappa_{n}^{1}\right\| \leq N \in \mathbb{N}$ for all $n=1,2, \ldots$

By the internal definition principle, extend the $\left(\kappa_{n}^{1}\right)_{n \in \mathbb{N}}$ to a hyperfinite increasing sequence $\left(\kappa_{n}^{1}\right)_{n=1}^{\omega}, \omega \in{ }^{*} \mathbb{N} \backslash \mathbb{N}$.

By the general saturation principle, there exists a hyperfinite $v \in{ }^{*} \mathbb{N} \backslash \mathbb{N}, v \leq \omega$, such that $\kappa_{\tau}^{1} \leq \kappa_{\tau+1}^{1}$ and $\left\|\kappa_{n+1}^{1}-\kappa_{n}^{1}\right\|=1$ for all $\tau \in{ }^{*} \mathbb{N}, \tau<v$.

Then $\left[\kappa_{0}^{1}-\left(\kappa_{v}^{1}-\kappa_{v-1}^{1}\right)\right]<\left[\kappa_{0}^{1}\right]$ and

$$
\left[\kappa_{n}^{1}\right] \leq\left[\kappa_{0}^{1}-\left(\kappa_{v}^{1}-\kappa_{v-1}^{1}\right)\right]
$$

for all $n=1,2, \ldots$, violating the condition $\left[\kappa_{0}^{1}\right]=\sup _{\tilde{X}}\left\{\left[\kappa_{n}^{1}\right]: n \in \mathbb{N}\right\}$. This contradiction completes the proof. 
We conclude this subsection with a brief discussion regarding the question on necessity of assumptions of Theorem 11. From one hand side if the positive cone $K_{X}$ in the OVS $\tilde{X}$ has the Levi property, then $K_{X}$ is normal (cf. [2, Thm.2.45.]). So, Theorem 9 ensures the normality of the positive cone $X_{+}$in $X$ provided by the condition 1) of Theorem 11. However, formally weaker condition 3) of $\sigma$-Dedekind completeness of $\tilde{X}$ along may not provide the normality of $X_{+}$in $X$. Therefore, the problem of necessity of the normality assumption of $X_{+}$in Theorem 11 remains open.

5.4. The following notion is motivated by the notion of a $(b)$-bounded set introduced in [3]. Given an ordered normed space $X$, consider the diagram

$$
X \hookrightarrow X^{\prime \prime} \hookrightarrow \ldots \hookrightarrow X^{(2 n)} \hookrightarrow \ldots
$$

of isometric embeddings. We say that a set $A \subset X$ is $X^{(2 n)}$-bounded if $A \subseteq[b, c]$ for some $b, c \in X^{(2 n)}$. Note that if $A$ is a $X^{(4)}$-bounded subset of a normed lattice $X$ then $|A| \leq c$ for some $c \in X_{+}^{(4)}$ The set $|A|^{\vee}$ of all finite suprema of elements of $|A|$ is upward directed and lies in the ball $B_{X^{\prime \prime}}(0,\|c\|)$ which is $w^{*}$-compact. Therefore $|A|^{\vee} \stackrel{w^{*}}{\rightarrow} a \in X^{\prime \prime}$ and since $X_{+}^{\prime \prime}$ is $w^{*}$-closed, $|A| \subseteq|A|^{\vee} \leq a$. Thus $A \in[-a, a]$ and hence $A$ is $X^{\prime \prime}$-bounded. The remark noted above is due to Süleyman Önal [23] and motivates the following conjecture.

Conjecture 1. Let $X$ be ordered Banach space with closed normal and generating cone $X_{+}$. Then every $X^{(4)}$-bounded set $A \subset X$ is $X^{\prime \prime}$-bounded.

As it was mentioned above, every Banach space $X^{(2 n)}$ can be embedded isometrically into $\tilde{X}$. Assume now that the cone $X_{+}$is normal and generating. Then, by Theorem 9, the isometric embeddings $X^{(2 n)} \hookrightarrow \tilde{X}$ become order embeddings. Therefore, 
any $X^{(2 n)}$-bounded subset of $X$ is $\tilde{X}$-bounded. Recall the aforementioned construction of isometric embedding of $X^{\prime \prime}$ into $\tilde{X}$. We take a hyperfinite dimensional internal subspace $V$ of ${ }^{*} X$ such that $X \subset V \subset{ }^{*} X$. Then $V$ is internally reflexive, that is $i(V)=V^{\prime \prime}$, where

$$
i(\phi)(\psi)=\psi(\phi) \quad(\forall \phi \in V)\left(\forall \psi \in V^{\prime}\right)
$$

So, one may identify all hyperfinite dimensional internal spaces $V^{(2 n)}$ with $V$ inductively as follows. Assume that $X^{(2 n)} \subset V^{(2 n)}=V$. The isometric embedding of $X^{(2(n+1))}$ into $V$ is given by $f \rightarrow \kappa_{f}$ for $f \in X^{(2(n+1))}$, where $\kappa_{f} \in V^{(2 n)}$ satisfies

$$
f(\xi)=\xi\left(\kappa_{f}\right) \quad\left(\xi \in X^{(2 n+1)}\right) .
$$

Thus we get an isometric and order embedding, say $i$, of $\bigcup_{n=0}^{\infty} X^{(2 n)}$ into $V$

$$
i: \bigcup_{n=0}^{\infty} X^{(2 n)} \hookrightarrow V \hookrightarrow{ }^{*} X .
$$

Since $\mu\left({ }^{*} X\right)$ is an order ideal by normality of $X_{+},(10)$ has natural factorization to the isometric and order embedding

$$
i: \bigcup_{n=0}^{\infty} X^{(2 n)} \hookrightarrow \tilde{X} .
$$

It can be shown that the embedding in (11) does not depend on choice of $V$. The following result is immediate consequence of (11).

Theorem 12. Let $X$ be ordered Banach space with closed normal and generating cone $X_{+}$and $n \in \mathbb{N}$. Then any

$X^{(2 n)}$-bounded subset of $X$ is $\tilde{X}$-bounded.

Theorem 12 motivates the following conjecture. 
Conjecture 2. Let $X$ be ordered Banach space with closed normal and generating cone $X_{+}$. Then every $\tilde{X}$-bounded set $A \subset X$ is $X^{\prime \prime}$-bounded.

Note that by Theorem 12, Conjecture 2 is formally stronger than Conjecture 1. 


\section{CHAPTER 6}

\section{LINEAR OPERATORS BETWEEN OVS}

Theorem 13. Let $X$ and $Y$ be ordered vector spaces such that $Y=Y_{+}-Y_{+}$, and $T$ : $X \rightarrow Y$ a linear operator. Then the following conditions are equivalent.

1) $T$ is order bounded;

2) ${ }^{*} T\left(\operatorname{fin}\left({ }^{*} X\right)\right) \subseteq \operatorname{fin}\left({ }^{*} Y\right)$;

3) ${ }^{*} T\left(\lambda\left({ }^{*} X\right)\right) \subseteq \lambda\left({ }^{*} Y\right)$;

4) ${ }^{*} T\left(\lambda\left({ }^{*} X\right)\right) \subseteq$ fin $\left({ }^{*} Y\right)$.

Proof. (1) $\Rightarrow(2)$ : Obvious.

$(2) \Rightarrow(3)$ : Let ${ }^{*} T\left(\operatorname{fin}\left({ }^{*} X\right)\right) \subseteq \operatorname{fin}\left({ }^{*} Y\right)$. Take an arbitrary $\kappa \in \lambda\left({ }^{*} X\right)$. Then, for some $u \in X, n \kappa \in[-u, u]$ for all $n \in \mathbb{N}$. Define $A_{n}=\left[-\frac{u}{n}, \frac{u}{n}\right]$. Then, the finite intersections of sets $A_{n}$ contain $\kappa$, i.e. are nonempty. Thus, by applying the general saturation principle, we can find $v \in{ }^{*} \mathbb{N} \backslash \mathbb{N}$ such that $\kappa \in\left[-\frac{u}{v}, \frac{u}{v}\right]$, that is, $v \kappa \in{ }^{*}[-u, u] \subseteq$ fin $\left({ }^{*} X\right)$, and by linearity of $\mathrm{T}, v^{*} T(\kappa)={ }^{*} T(v \kappa) \in \operatorname{fin}\left({ }^{*} Y\right)$ by hypothesis. Since $-v \kappa \leqslant-n \kappa \leqslant n \kappa \leqslant$ $v \kappa$, we have ${ }^{*} T(\kappa) \in \lambda\left({ }^{*} Y\right)$. Therefore, ${ }^{*} T\left(\lambda\left({ }^{*} X\right)\right) \subseteq \lambda\left({ }^{*} Y\right)$.

$(3) \Rightarrow(4)$ : Obvious.

$(4) \Rightarrow(2)$ : Assume that ${ }^{*} T(\kappa)$ is not an element of $\operatorname{fin}\left({ }^{*} Y\right)$ for some $\kappa \in$ fin ${ }^{*} X$. For every $n \in \mathbb{N}$ and every $u \in Y_{+}$, assign

$$
A_{n, u}:=\left\{k \in{ }^{*} \mathbb{N}: k \geqslant n \wedge^{*} T\left(k^{-1} \kappa\right) \notin[-u, u]\right\}
$$

By construction, the sets $A_{n, u}$ are nonempty, internal and satisfy the finite intersection 
property, since

$$
A_{\max (n, p), u+v} \subseteq A_{n, u} \cap A_{p, v}
$$

for arbitrary $n, p \in \mathbb{N}$ and $u, v \in Y_{+}$. By applying the general saturation principle, we find $v \in \bigcap_{n, u} A_{n, u}$. Obviously, $v \in{ }^{*} \mathbb{N} \backslash \mathbb{N}$, and thus $v^{-1} \kappa \in \lambda\left({ }^{*} X\right)$. Hence, ${ }^{*} T\left(v^{-1} \kappa\right) \in$ fin $\left({ }^{*} Y\right)$, that is, there exist $z \in Y_{+}$such that ${ }^{*} T\left(v^{-1} \kappa\right) \in[-z, z]$. A contradiction since $v \in A_{1, z}$. Hence the claim.

$(2) \Rightarrow(1):$ Take an arbitrary $u \in X_{+}$. By hypothesis

$$
{ }^{*}(T([-u, u]))={ }^{*} T\left({ }^{*}[-u, u]\right) \subseteq \operatorname{fin}\left({ }^{*} Y\right)
$$

Hence, by Theorem 1 , the set $T([-u, u])$ is order bounded. 


\section{REFERENCES}

[1] Albeverio S., Høegh-Krohn R., Fenstad J. E., and Lindstrøm T. Nonstandard methods in stochastic analysis and mathematical physics. Pure and Applied Mathematics. Vol.122, Academic Press Inc. (1986).

[2] Aliprantis C. D. and Tourky R. Cones and duality. Graduate Studies in Mathematics. Vol.84, American Mathematical Society. (2007).

[3] Alpay S., Altin B, and Tonyali C. On property (b) of vector lattices. Positivity 2003; $7:$ 135-139.

[4] Davis M. Applied nonstandard analysis. Pure and Applied Mathematics. (1977).

[5] Emel'yanov E. Yu. Infinitesimals in ordered vector spaces. Vladikavkaz. Mat. Zh. 2013; 15. (No.1): 18-22.

[6] Emel'yanov E. Yu. Erratum to“Infinitesimals in ordered vector spaces". Vladikavkaz. Mat. Zh. 2013; 15. (No.2): 82-83.

[7] Emel'yanov E. Yu. Infinitesimals in vector lattices. Math. Appl., Kluwer Acad. Publ., Dordrecht, 2000; 525: 161-230.

[8] Emel'yanov E. Yu. Invariant homomorphisms of nonstandard extensions of Boolean algebras and vector lattices. Sibirsk. Mat. Zh. 1997; 38: 286-296. (English translation: Siberian Math. J. 1997; 38: 244-252.) 
[9] Emel'yanov E. Yu. Infinitesimal analysis and vector lattices. Siberian Adv. Math. 1996; 6: 19-70.

[10] Emel'yanov E. Yu. An infinitesimal approach to the representation of vector lattices by spaces of continuous functions on a compactum. Dokl. Akad. Nauk 1995; 344. (No.1): 9-11.

[11] Emel'yanov E. Yu. Banach-Kantorovich spaces associated with order-hulls of decomposable lattice-normed spaces. Sibirsk. Mat. Zh. 1995; 36: 72-85. (English translation: Siberian Math. J. 1995; 36: 66-77.)

[12] Emel'yanov E. Yu. Order hulls of vector lattices. Dokl. Akad. Nauk. 1995; 340. (No.3): 303-304.

[13] Emel'yanov E. Yu. Ordered and regular hulls of vector lattices. Sibirsk. Mat. Zh. 1994; 35: 1243-1252. (English translation: Siberian Math. J. 1995; 35: 11011108.)

[14] Emel'yanov E. Yu. Nonstandard hulls of vector lattices. Sibirsk. Mat. Zh. 1994; 35: 83-95. (English translation: Siberian Math. J. 1994; 35: 77-87.)

[15] Emel'yanov E. Yu. and Gül H. Nonstandard hulls of ordered vector spaces. Positivity, Springer-Birkhäuser ; DOI: 10.1007/s11117-015-0364-2 . (2015)

[16] Gorokhova S. G. and Emel'yanov E. Yu. On the concept of stability of order convergence in vector lattices. Sibirsk. Mat. Zh. 1994; 35: 1026-1031. (English translation: Siberian Math. J. 1994; 35: 912-916.) 
[17] Henson C. W. and Moore L. C. Nonstandard analysis and the theory of Banach spaces. (Nonstandard analysis-recent developments, Victoria, B.C. - 1980) Lecture Notes in Math. Vol.983, Springer, Berlin. (1983).

[18] Hurd A. and Loeb P. A. Introduction to Nonstandard Real Analysis. Academic Press, New York. (1985).

[19] Keisler H.J. Foundations of Infinitesimal Calculus. Prindle, Weber and Schmidt, Boston. (1976).

[20] Kusraev A. G. and Kutateladze S. S. Nonstandard methods of analysis. Mathematics and its Applications. Vol.291, Kluwer Academic Publishers Group, Dordrecht. (1994).

[21] Luxemburg W. A. J. A general theory of monads. (Applications of Model Theory to Algebra, Analysis, and Probability, Internat. Sympos., Pasadena, Calif. (1967)), Holt, Rinehart and Winston. (1969).

[22] Luxemburg W. A. J. and Zaanen A. C. Riesz Spaces I. North-Holland: Amsterdam. (1971).

[23] Onal, S. Private communication. (2013)

[24] Luxemburg W. A. J. and Stroyan K. D. Introduction to the theory of infinitesimals. Pure and Applied Mathematics. No.72, Academic Press. (1976).

[25] Robinson A. Nonstandard Analysis. North-Holland Publ., Amsterdam. (1966).

[26] Schaefer H. H. and Wolff M. P. Topological vector spaces. Graduate Texts in Mathematics. Vol.3, Second edition. Springer-Verlag, New York. (1999). 
[27] Veksler A. I. Archimedean principle in homomorphic images of $l$-groups and of vector lattices. Izv. Vys. Ucebn. Zaved. Matematika. 1966; 54. (No.4): 33-38.

[28] Vulikh B. Z. Introduction to Theory of Partially Ordered Spaces. Groningen, the Netherlands: Noordhoff. (1967).

[29] Zaanen A. C. Riesz Spaces II. North-Holland, Amsterdam. (1983). 


\section{APPENDIX A}

\section{THE ULTRAPOWER CONSTRUCTION OF NONSTANDARD EXTENSION}

\section{A.1 Infinitesimals}

- A filter $\mathscr{F}$ on $\mathbb{N}$ is a set $\mathscr{F}$ of subsets of $\mathbb{N}$ such that

$\emptyset \notin \mathscr{F}, \quad \mathbb{N} \in \mathscr{F}$

. If $A, B \in \mathscr{F}$, then $A \cap B \in \mathscr{F}$

$\cdot A \in \mathscr{F}$ and $A \subseteq B \Rightarrow B \in \mathscr{F}$

- A filter $\mathscr{F}$ on $\mathbb{N}$ is called free if it does not contain any finite set.

- A filter $\mathscr{U}$ is called an ultrafilter over $\mathbb{N}$ if for all $E \subseteq \mathbb{N}$ either $E \in \mathscr{U}$ or $\mathbb{N} \backslash E \in$ $\mathscr{U}$.

- Ultrafilter Theorem There exist free ultrafilters $\mathscr{U}$ on $\mathbb{N}$ extending the filter of cofinite sets.

Let $\mathscr{U}$ be a free ultrafilter on $\mathbb{N}$ and introduce an equivalence relation on sequences in $\mathbb{R}^{\mathbb{N}}$ as

$$
f \sim_{\mathscr{U}} g \text { iff }\{n \in \mathbb{N}: f(n)=g(n)\} \in \mathscr{U}
$$

$\mathbb{R}^{\mathbb{N}}$ divided out by the equivalence relation $\sim \mathscr{U}$ gives us the nonstandart extension ${ }^{*} \mathbb{R}$, the hyperreals; in symbols ,

$$
{ }^{*} \mathbb{R}=\mathbb{R}^{\mathbb{N}} / \mathscr{U}
$$

If $f \in \mathbb{R}^{\mathbb{N}}$, we denote its image in ${ }^{*} \mathbb{R}$ by $f_{\mathscr{U}}$. 
For any real number $r \in \mathbb{R}$, let $\mathbf{r}$ denote the constant function with value $r$ in $\mathbb{R}^{\mathbb{N}}$, i.e., $\mathbf{r}(n)=r$ for all $n \in \mathbb{N}$. Then we have a natural embedding

$$
{ }^{*}: \mathbb{R} \rightarrow{ }^{*} \mathbb{R}
$$

by setting ${ }^{*} r=r_{\mathscr{U}}$, for all $r \in \mathbb{R}$.

As an algebraic structure, $\mathbb{R}$ is a complete ordered field, i.e., a structure of the form

$$
\langle\mathbb{R},+, \cdot,<, 0,1\rangle
$$

The ${ }^{*}$-embedding sends 0 to ${ }^{*} 0=\mathbf{0}_{\mathscr{U}}$ and 1 to ${ }^{*} 1=\mathbf{1}_{\mathscr{U}}$. Also, we have

$$
f_{\mathscr{U}}=g_{\mathscr{U}} \text { iff }\{n \in \mathbb{N}: f(n)=g(n)\} \in \mathscr{U} .
$$

Similarly, we extend $<$ to $* \mathbb{R}$ by setting

$$
f_{\mathscr{U}}<g_{\mathscr{U}} \text { iff }\{n \in \mathbb{N}: f(n)<g(n)\} \in \mathscr{U} \text {. }
$$

We now have a linear oeder on ${ }^{*} \mathbb{R}$ and can verify that ${ }^{*} \mathbb{R}$ contains infinitesimals and infinite numbers. A (positive) infinitesimal $\delta$ in ${ }^{*} \mathbb{R}$ is an element $\delta \in{ }^{*} \mathbb{R}$ such that ${ }^{*} 0<\delta<{ }^{*} r$ for all $r>0$ in $\mathbb{R}$.

Infinitesimals exist; let $f(n)=1 / n$ for all $n \in \mathbb{N}$. Then $\delta=f_{\mathscr{U}}$ is a positive infinitesimal. Also notice that $g(n)=1 / n^{2}$ defines another infinitesimal, say $\delta^{\prime}$ and that $\delta^{\prime}<\delta$ in ${ }^{*} \mathbb{R}$.

In the same way $h(n)=n$ and $p(n)=n^{2}$ introduce infinite numbers, say $\omega=h_{\mathscr{U}}$ and $\omega^{\prime}=p_{\mathscr{U}}$, and $\omega<\omega^{\prime}$ in ${ }^{*} \mathbb{R}$.

It remains to extend the operations + and $\cdot$ to $* \mathbb{R}$. Set

$$
f_{\mathscr{U}}+g_{\mathscr{U}}=h_{\mathscr{U}} \text { iff }\{n \in \mathbb{N}: f(n)+g(n)=h(n)\} \in \mathscr{U} \text {. }
$$




$$
f_{\mathscr{U}} \cdot g_{\mathscr{U}}=h_{\mathscr{U}} \text { iff }\{n \in \mathbb{N}: f(n) \cdot g(n)=h(n)\} \in \mathscr{U} \text {. }
$$

Let $F$ be an $n$-ary function on $\mathbb{R}$, i.e.,

$$
F: \mathbb{R} \times \cdots \times \mathbb{R} \rightarrow \mathbb{R}
$$

We introduce the extended function ${ }^{*} F$ by the equivalence

$$
{ }^{*} F\left(f_{\mathscr{U}}^{1}, \ldots, f_{\mathscr{U}}^{n}\right)=g_{\mathscr{U}} \text { iff }\left\{i \in \mathbb{N}: F\left(f^{1}(i), \ldots, f^{n}(i)\right)\right\} \in \mathscr{U}
$$

The structure $\langle\mathbb{R},+, \cdot,<,|\cdot|, 0,1\rangle$ has an associated simple language $L(\mathbb{R})$ that can be used to describe the kind of properties of $\mathbb{R}$ that are preserved under the embedding $*: \mathbb{R} \rightarrow{ }^{*} \mathbb{R}$.

The elementary formulas of $L(\mathbb{R})$ are expressions of the form

$$
\text { (i) } t_{1}+t_{2}=t_{3} \text { (ii) } t_{1} \cdot t_{2}=t_{3} \text { (iii) }\left|t_{1}\right|=t_{2} \text { (iv) } t_{1}=t_{2} \text { (v) } t_{1}<t_{2} \text { (vi) } t_{1} \in X \text {, }
$$

where $t_{1}, t_{2}, t_{3}$ are either the constants 0 or 1 or a variable for an arbitrary number $r \in \mathbb{R}$ and $X$ is a variable for a subset $A \subseteq \mathbb{R}$.

From the elementary formulas we generate the class of all formulas or expressions of $L(\mathbb{R})$ using the propositional connectives

$$
\wedge \text { (and), } \vee(\text { or }), \neg \text { (not), } \rightarrow \text { (if, then) }
$$

and the number quantifiers

$$
\forall x \text { for all } \mathrm{x} \text { (in } \mathbb{R}), \exists x \text { for some } \mathrm{x}(\text { in } \mathbb{R} \text { ) }
$$

by the rules : 
(vii) If $\Phi$ and $\Psi$ are formulas of $L(\mathbb{R})$, then

$$
\Phi \wedge \Psi, \Phi \vee \Psi, \neg \Phi, \Phi \rightarrow \Psi
$$

are formulas of $L(\mathbb{R})$.

(viii) If $\Phi$ is a formula of $L(\mathbb{R})$ and $\mathrm{x}$ is a number variable, then $\forall x \Phi$ and $\exists x \Phi$ are formulas of $L(\mathbb{R})$.

Every formula in $L(\mathbb{R})$ has an immediate meaning or interpretation in the structure $\mathbb{R}$ and can also be interpreted in the extended structure $* \mathbb{R}$.

We can now state the main result about ultrafilter extensions, which has the general transfer principle as immediate corollary.

- Theorem of Loś Let $\Phi\left(X_{1}, \ldots, X_{m}, x_{1}, \ldots, x_{n}\right)$ be a formula of $L(\mathbb{R})$. Then for any $A_{1}, \ldots, A_{m} \subseteq \mathbb{R}$ and $f_{\mathscr{U}}^{1}, \ldots, f_{\mathscr{U}}^{n} \in{ }^{*} \mathbb{R}$,

$$
\Phi\left({ }^{*} A_{1}, \ldots,{ }^{*} A_{m}, f_{\mathscr{U}}^{1}, \ldots, f_{\mathscr{U}}^{n}\right) \text { iff }\left\{i \in \mathbb{N}: \Phi\left(A_{1}, \ldots, A_{m}, f_{\mathscr{U}}^{1}(i), \ldots, f_{\mathscr{U}}^{n}(i)\right\} \in \mathscr{U}\right.
$$

- Transfer Principle Let $\Phi\left(X_{1}, \ldots, X_{m}, x_{1}, \ldots, x_{n}\right)$ be a formula of $L(\mathbb{R})$. Then for any $A_{1}, \ldots, A_{m} \subseteq \mathbb{R}$ and $r_{1}, \ldots, r_{n} \in \mathbb{R}, \Phi\left(A_{1}, \ldots, A_{m}, r_{1}, \ldots, r_{n}\right)$ is true in $\mathbb{R}$ iff $\Phi\left({ }^{*} A_{1}, \ldots,{ }^{*} A_{m},{ }^{*} r_{1}, \ldots,{ }^{*} r_{n}\right)$ is true in ${ }^{*} \mathbb{R}$.

\section{A.2 The extended universe}

The structure $\mathbb{R}$ is not a large enough domain for the development of classical mathematics. We need an extended universe that, in addition to numbers and functions, also contains sets of functions, sets of spaces of functions, etc. In more generality, given any set $\mathrm{S}$ we introduce the superstructure $\mathrm{V}(\mathrm{S})$ over $\mathrm{S}$ as follows.

$$
V_{1}(S):=S
$$




$$
\begin{aligned}
V_{n+1}(S) & :=\mathscr{P}\left(V_{n}(S)\right), \\
V(S) & :=\bigcup_{n} V_{n}(S) .
\end{aligned}
$$

Classical analysis lives inside $V(\mathbb{R})$. The extended universe of nonstandart analysis will be obtained by postulating an extension $* \mathbb{R} \supseteq \mathbb{R}$ and postulating an embedding

$$
{ }^{*}: V(\mathbb{R}) \rightarrow V\left({ }^{*} \mathbb{R}\right)
$$

that will have properties similar to the embedding ${ }^{*}: \mathbb{R} \rightarrow{ }^{*} \mathbb{R}$ constructed in Section 1 . First of all, we assume the following principle.

- Extension Principle ${ }^{*} \mathbb{R}$ is a proper extension of $\mathbb{R}$ and ${ }^{*} r=r$ for all $r \in \mathbb{R}$.

In the model of Section 1 , this means we identify $\mathbb{R}$ with its ${ }^{*}$-image in ${ }^{*} \mathbb{R}$. We shall now extend the ultrafilter construction to demonstrate that superstructure embeddings of the type above satisfying a transfer principle analogous to the one in Section 1 exist.

I. Constructing the bounded ultrapower A sequence $\left\langle A_{1}, A_{2}, \ldots\right\rangle$ of elements of $V(\mathbb{R})$ is bounded if there is a fixed n such that each $A_{i} \in V_{n}(\mathbb{R})$. Two bounded sequence $\mathrm{A}$ and $\mathrm{B}$ are equivalent with respect to free ultrafilter $\mathscr{U}$, in symbols $A \sim_{\mathscr{U}} B$ iff $\left\{i \in \mathbb{N}: A_{i}=B_{i}\right\} \mathscr{U}$. We let $A_{\mathscr{U}}$ denote the equivalence class of A and define the bounded ultrapower by

$$
V(\mathbb{R})^{\mathbb{N}} / \mathscr{U}=\left\{A_{\mathscr{U}}: A \text { is a bounded } V(\mathbb{R}) \text {-sequence }\right\}
$$

We define the membership relation $\in_{\mathscr{U}}$ in the ultrapower by

$$
A_{\mathscr{U}} \in \mathscr{U} B_{\mathscr{U}} \quad \text { iff }\left\{i \in \mathbb{N}: A_{i} \in B_{i}\right\} \in \mathscr{U}
$$

There is a natural proper embedding

$$
i: V(\mathbb{R}) \rightarrow V(\mathbb{R})^{\mathbb{N}} / \mathscr{U}
$$


namely let $i(A)=\langle A, A, \ldots\rangle_{\mathscr{U}}$ the equivalence class corresponding to constant sequence.

II. Embedding $V(\mathbb{R})^{\mathbb{N}} / \mathscr{U}$ into $V\left({ }^{*} \mathbb{R}\right)$

${ }^{*} \mathbb{R}$ is the (bounded) ultrapower $\mathbb{R}^{\mathbb{N}} / \mathscr{U}$. But $V(\mathbb{R})^{\mathbb{N}} / \mathscr{U}$ will not be the same as the full superstructure $V\left({ }^{*} \mathbb{R}\right)$. We shall now construct an embedding

$$
j: V(\mathbb{R})^{\mathbb{N}} / \mathscr{U} \rightarrow V\left({ }^{*} \mathbb{R}\right)
$$

such that (i) $j$ is the identity on ${ }^{*} \mathbb{R}$ and (ii) if $A_{\mathscr{U}} \notin{ }^{*} \mathbb{R}$, then $j\left(A_{\mathscr{U}}\right)=\left\{j\left(B_{\mathscr{U}}\right): B_{\mathscr{U}} \in \mathscr{U}\right.$ $\left.A_{\mathscr{U}}\right\}$. This means that the relation $\in_{\mathscr{U}}$ in the ultrapower is mapped into the ordinary membership relation $V\left({ }^{*} \mathbb{R}\right)$.

The embedding $j$ is constructed in stages. Let

$$
V_{k}(\mathbb{R})^{\mathbb{N}} / \mathscr{U}=\left\{A_{\mathscr{U}}: A \text { is a sequence from } V_{k}(\mathbb{R})\right\} .
$$

Then the bounded ultrapower is the union of the chain

$$
{ }^{*} \mathbb{R}=V_{1}(\mathbb{R})^{\mathbb{N}} / \mathscr{U} \subseteq \ldots \subseteq V_{k}(\mathbb{R})^{\mathbb{N}} / \mathscr{U} \subseteq \ldots,
$$

and we can define $j$ by induction. For $k=1, j$ must be identity. If $A_{\mathscr{U}} \in V_{k+1}(\mathbb{R})^{\mathbb{N}} / \mathscr{U}$ and $A_{\mathscr{U}} \notin^{*} \mathbb{R}$, we simply set $j\left(A_{\mathscr{U}}\right)=\left\{j\left(B_{\mathscr{U}}\right): B_{\mathscr{U}} \in \mathscr{U}_{\mathscr{U}} A_{\mathscr{U}}\right\}$. This makes sense : if $B_{\mathscr{U}} \in \mathscr{U} A_{\mathscr{U}}$ it follows from the definition that $\left\{i \in \mathbb{N}: B_{i} \in V_{k}(\mathbb{R})\right\} \in \mathscr{U}$, i.e., $B_{\mathscr{U}} \in V_{k}(\mathbb{R})^{\mathbb{N}} / \mathscr{U}$, which means that $j\left(B_{\mathscr{U}}\right)$ is defined at a previous stage of the inductive construction.

Combining $i$ and $j$ we get a model of the extended nonstandart universe where ${ }^{*} A=j(i(A))$ for any $A \in V(\mathbb{R})$.

Here $V(\mathbb{R})$ and $V\left({ }^{*} \mathbb{R}\right)$ are connected by a transfer principle generalizing the one in Section 1 . The structure $\mathbb{R}$ has an associated elementary language $L(\mathbb{R})$ which we used to give the necessary precision to the transfer principle. We need a similar formal tool to state the extended transfer principle. 
The language $L(V(\mathbb{R}))$ will be an extension of the language $L(\mathbb{R})$. We add to our stock of elementary formulas in Section 1 expressions of the form " $X \in Y$ " where $X$ and $Y$ are variables for arbitrary sets in $V(\mathbb{R})$.

We keep the logical symbols of $L(\mathbb{R})$ but in addition to the number quantifiers we add bounded set quantifiers

$$
\begin{gathered}
\forall X \in Y \text { for all sets } \mathrm{X} \text { element of } \mathrm{Y}, \\
\exists X \in Y \text { for some set } \mathrm{X} \text { element of } \mathrm{Y} \text {. }
\end{gathered}
$$

Formulas $\Phi$ of $L(V(\mathbb{R}))$ are then constructed in exactly the same way as formulas of $L(\mathbb{R})$ and can be interpreted in a natural way in any of the structures $V(\mathbb{R}), V(\mathbb{R})^{\mathbb{N}} / \mathscr{U}$ and $V\left({ }^{*} \mathbb{R}\right)$.

- Transfer Principle Let $A_{1}, \ldots, A_{n} \in V(\mathbb{R})$. Any $L(V(\mathbb{R}))$ statement $\Phi$ that is true of $A_{1}, \ldots, A_{n}$ in $V(\mathbb{R})$ is true of ${ }^{*} A_{1}, \ldots,{ }^{*} A_{n}$ in $V\left({ }^{*} \mathbb{R}\right)$, and conversely.

- Let $A \in V\left({ }^{*} \mathbb{R}\right)$, then

(i) A is called standard if $A={ }^{*} B$ for some $B \in V(\mathbb{R})$,

(ii) A is called internal if $A \in{ }^{*} B$ for some $B \in V(\mathbb{R})$,

(iii) A is called external if $A$ is not internal.

- Internal Definition Principle Let $A_{1}, \ldots, A_{n}$ be internal sets in $V\left({ }^{*} \mathbb{R}\right)$ and let $\Phi\left(X_{1}, \ldots, X_{n}, x\right)$ be an $L(V(\mathbb{R}))$ statement. Then the set

$$
\left\{x \in A_{i}: \Phi\left(X_{1}, \ldots, X_{n}, x\right)\right\}
$$

is internal.

- Remark We have restricted ourselves to $V(\mathbb{R})$, in some cases it may be more natural to work inside a different superstructure. For instance, let $\mathrm{E}$ be a linear normed space over the complex numbers $\mathbb{C}$. It will be natural to work in the superstructure 
$V(E \cup \mathbb{C}$, i.e., to regard $\mathrm{E}$ and $\mathbb{C}$ as basic objects. The rest would be a set-theoretic construction from the set of "urelements" $E \cup \mathbb{C}$.

- General Saturation Principle Let $\kappa$ be an infinite cardinal. A nonstandard extension is called $\kappa$-saturated if for every family $\left\{X_{i}\right\}_{i \in I}, \operatorname{card}(I)<\kappa$ with the finite intersection property, the intersection $\bigcap_{i \in I} X_{i}$ is nonempty.

- The uniqueness theorem for superstructure embeddings There is up to isomorphism a unique superstructure embedding ${ }^{*}: V(\mathbb{R}) \rightarrow V\left({ }^{*} \mathbb{R}\right)$ such that ${ }^{*}$ satisfies the transfer principle and is saturated in the sense above [19] .

We have borrowed much of the above presentation from [1]. 


\section{VITA}

\section{HASAN GÜL}

e032209@metu.edu.tr

\section{EDUCATION}

M.S. Mathematics, University of Illinois at Urbana-Champaign, IL, USA 1991

B.S Chemistry, $\quad$ Middle East Technical University, Ankara 1985

High School Diploma, Ankara Fen Lisesi, Ankara 1981

Bursa Maarif Koleji, Bursa 1978

\section{WORK EXPERIENCE}

Part-time Instructor

Middle East Technical University, Ankara 9/2001 to 06/2003

Maintained a teaching load of two undergraduate courses each semester.

\section{Instructor}

Middle East Technical University, Ankara $\quad$ 09/1992 to 09/1999

Taught two undergraduate courses each semester during the allocated seven years for the position (Araştırma Görevlisi kadrosunda Öğretim Görevlisi)

\section{Teaching Assistant}

University of Illinois at Urbana-Champaign, Urbana, IL $\quad$ 09/1988 to 2/1992

Middle East Technical University, Ankara $\quad$ 09/1986 to 09/1992

Ohio State University, Columbus, OH, USA $\quad 09 / 1985$ to 06/1986

\section{PUBLICATIONS}

Emelyanov E. and Gül H. Nonstandard hulls of ordered vector spaces, Positivity, Springer-Birkhauser ; DOI: 10.1007/s11117-015-0364-2 .(2015)

Gül H. A General Approach to Radius Ratios of Simple Ionic Crystals, Journal of Chemical .Education, May 1985 